\title{
A New Positive Definite Expanded Mixed Finite Element Method for Parabolic Integrodifferential Equations
}

\author{
Yang Liu, ${ }^{1}$ Hong Li, ${ }^{1}$ Jinfeng Wang, $^{2}$ and Wei Gao ${ }^{1}$ \\ ${ }^{1}$ School of Mathematical Sciences, Inner Mongolia University, Hohhot 010021, China \\ ${ }^{2}$ School of Statistics and Mathematics, Inner Mongolia Finance and Economics College, \\ Hohhot 010070, China
}

Correspondence should be addressed to Hong Li, smslh@imu.edu.cn

Received 4 February 2012; Revised 3 April 2012; Accepted 19 April 2012

Academic Editor: Jinyun Yuan

Copyright (C) 2012 Yang Liu et al. This is an open access article distributed under the Creative Commons Attribution License, which permits unrestricted use, distribution, and reproduction in any medium, provided the original work is properly cited.

\begin{abstract}
A new positive definite expanded mixed finite element method is proposed for parabolic partial integrodifferential equations. Compared to expanded mixed scheme, the new expanded mixed element system is symmetric positive definite and both the gradient equation and the flux equation are separated from its scalar unknown equation. The existence and uniqueness for semidiscrete scheme are proved and error estimates are derived for both semidiscrete and fully discrete schemes. Finally, some numerical results are provided to confirm our theoretical analysis.
\end{abstract}

\section{Introduction}

In this paper, we consider the following initial-boundary value problem of parabolic partial integrodifferential equations:

$$
\begin{gathered}
u_{t}-\nabla \cdot\left(a(x, t) \nabla u+b(x, t) \int_{0}^{t} \nabla u d s\right)=f(x, t), \quad(x, t) \in \Omega \times J, \\
u(x, t)=0, \quad(x, t) \in \partial \Omega \times \bar{J}, \\
u(x, 0)=u_{0}(x), \quad x \in \Omega,
\end{gathered}
$$

where $\Omega$ is a bounded convex polygonal domain in $\mathbb{R}^{d},(d=1,2,3)$ with a smooth boundary $\partial \Omega, J=(0, T]$ is the time interval with $0<T<\infty$. The coefficients $a=a(x, t), b=b(x, t)$ are 
two functions, which satisfy the property that there exist some positive constants $a_{\min }, a_{\max }$, $b_{\min }$, and $b_{\max }$ such that $0<a_{\min } \leq a(x, t) \leq a_{\max }$ and $0<b_{\min } \leq b(x, t) \leq b_{\max }$.

Parabolic integrodifferential equations are a class of very important evolution equations which describe many physical phenomena such as heat conduction in material with memory, compression of viscoelastic media, and nuclear reactor dynamics. In recent years, a lot of researchers have studied the numerical methods for parabolic integrodifferential equations, such as finite element methods [1-5], mixed finite element methods [6-9], and finite volume element method [10] and so forth.

In 1994, Chen [11, 12] proposed a new mixed method, which is called a expanded mixed finite element method and proved some mathematical theories for second-order linear elliptic equation. Compared to standard mixed element methods, the expanded mixed method is expanded in the sense that three variables are explicitly approximated, namely, the scalar unknown, its gradient, and its flux (the tensor coefficient times the gradient). In 1997, Arbogast et al. [13] derived and exploited a connection between the expanded mixed method and a certain cell-centered finite difference method. And Chen proved some mathematical theories for second-order quasilinear elliptic equation [14] and fourth-order elliptic problems [15]. With the development of the expanded mixed finite element method, the method was applied to many evolution equations. In [16], some error estimates of the expanded mixed element for a kind of parabolic equation were given. Woodward and Dawson [17] studied the expanded mixed finite element method for nonlinear parabolic equation. Wu and $\mathrm{Chen}$ et al. [18-22] studied the two-grid methods for expanded mixed finite-element solution of semilinear reaction-diffusion equations. Song and Yuan [23] proposed the expanded upwindmixed multistep method for the miscible displacement problem in three dimensions. Guo and Chen [24] developed and analysed an expanded characteristic-mixed finite element method for a convection-dominated transport problem. In 2010, Chen and Wang [25] proposed an $H^{1}$-Galerkin expanded mixed method for a nonlinear parabolic equation in porous medium flow, and Liu and Li [26] studied the $H^{1}$-Galerkin expanded mixed method for pseudohyperbolic equation. Liu [27], studied the $H^{1}$-Galerkin expanded mixed method for RLWBurgers equation and proved semidiscrete and fully discrete optimal error estimates. Jiang and $\mathrm{Li}$ [28] studied an expanded mixed semidiscrete scheme for the problem of purely longitudinal motion of a homogeneous bar. In [29, 30], the expanded mixed covolume method was studied for the linear integrodifferential equation of parabolic type and elliptic problems, respectively. In [31], a posteriori error estimator for expanded mixed hybrid methods was studied and analysed.

In 2001, Yang [32] proposed a new mixed finite element method called the splitting positive definite mixed finite element procedure to treat the pressure equation of parabolic type in a nonlinear parabolic system describing a model for compressible flow displacement in a porous medium. Compared to standard mixed methods whose numerical solutions have been quite difficult because of losing positive definite properties, the proposed one does not lead to some saddle point problems. From then on, the method was applied to the hyperbolic equations [33] and pseudo-hyperbolic equations [34].

In this paper, our purpose is to propose and analyse a new expanded mixed method based on the positive definite system [32-34] for parabolic integrodifferential equations. Compared to expanded mixed methods, the proposed mixed element system is symmetric positive definite and avoids some saddle point problems. What is more, both the gradient equation and the flux equation are separated from its scalar unknown equation. The existence and uniqueness for semidiscrete scheme are proved and error estimates are derived for both semidiscrete and fully discrete schemes. 
The layout of the paper is as follows. In Section 2, the positive definite expanded mixed weak formulation and semidiscrete mixed scheme are formulated, and the proof of the existence and uniqueness of the discrete solutions is given. Error estimates are derived for both semidiscrete and fully discrete schemes for problems, respectively, in Sections 3 and 4. In Section 5, some numerical results are provided to illustrate the efficiency of our method. Finally in Section 6, we will give some concluding remarks about the positive definite expanded mixed finite element method.

Throughout this paper, $C$ will denote a generic positive constant which does not depend on the spatial mesh parameters $h_{u}, h_{\sigma}$ and time-discretization parameter $\delta$ and may be different at their occurrences. Usual definitions, notations, and norms of the Sobolev spaces as in [35-37] are used. We denote the natural inner product in $L^{2}(\Omega)$ or $\left[L^{2}(\Omega)\right]^{d}$ by $(\cdot, \cdot)$ with norm $\|\cdot\|_{L^{2}(\Omega)}$ or $\|\cdot\|_{L^{2}(\Omega)}$ and introduce the function space $\mathbf{W}=H(\operatorname{div} ; \Omega)=\{\omega \in$ $\left.\left[L^{2}(\Omega)\right]^{d} ; \nabla \cdot \omega \in L^{2}(\Omega)\right\}$.

\section{A New Expanded Mixed Variational Formulation}

Introducing the auxiliary variables:

$$
\lambda=\nabla u, \quad \sigma=a(x, t) \nabla u+b(x, t) \int_{0}^{t} \nabla u d s=a \lambda+b \int_{0}^{t} \lambda d s,
$$

Then we obtain the equivalent system of parabolic partial integrodifferential equations for the problem (1.1):

$$
\begin{aligned}
& \text { (a) } u_{t}-\nabla \cdot \sigma=f(x, t), \quad(x, t) \in \Omega \times J \\
& \text { (b) } \lambda-\nabla u=0, \quad(x, t) \in \Omega \times J, \\
& \text { (c) } \sigma-a \curlywedge-b \int_{0}^{t} \lambda d s=0, \quad(x, t) \in \Omega \times J
\end{aligned}
$$

with the initial values $\lambda(x, 0)=\nabla u_{0}(x), \sigma(x, 0)=a \nabla u_{0}(x)$, and $u(x, 0)=u_{0}(x)$.

Then, the following expanded mixed weak formulation of (2.2) can be given by:
(a) $\left(u_{t}, v\right)-(\nabla \cdot \sigma, v)=(f(x, t), v), \quad \forall v \in L^{2}(\Omega)$,
(b) $(\lambda, \mathbf{w})+(u, \nabla \cdot \mathbf{w})=0, \quad \forall \mathbf{w} \in \mathbf{W}$,
(c) $(\sigma, \mathbf{z})-(a \lambda, \mathbf{z})-\left(b \int_{0}^{t} \lambda d s, \mathbf{z}\right)=0, \quad \forall \mathbf{z} \in \mathbf{W}$.

From (2.3) (b) we derive

$$
\left(\lambda_{t}, \mathbf{w}\right)+\left(u_{t}, \nabla \cdot \mathbf{w}\right)=0 .
$$


Taking $v=\nabla \cdot \mathbf{w}$ in (2.3)(a) for $\mathbf{w} \in \mathbf{W}$ and then substituting it into (2.4), we derive a new equivalent expanded mixed weak formulation of the system (2.3):

$$
\begin{aligned}
& \text { (a) }\left(\lambda_{t}, \mathbf{w}\right)+(\nabla \cdot \sigma, \nabla \cdot \mathbf{w})=-(f(x, t), \nabla \cdot \mathbf{w}), \quad \forall \mathbf{w} \in \mathbf{W}, \\
& \text { (b) }(\sigma, \mathbf{z})-(a \lambda, \mathbf{z})-\left(b \int_{0}^{t} \lambda d s, \mathbf{z}\right)=0, \quad \forall \mathbf{z} \in \mathbf{W}, \\
& \text { (c) }\left(u_{t}, v\right)-(\nabla \cdot \sigma, v)=(f(x, t), v), \quad \forall v \in L^{2}(\Omega) .
\end{aligned}
$$

Let $\tau_{h_{u}}$ and $\tau_{h_{\sigma}}$ be two families of quasi-regular partitions of the domain $\Omega$, which may be the same one or not, such that the elements in the partitions have the diameters bounded by $h_{u}$ and $h_{\sigma}$, respectively. Let $X_{h_{u}} \subset L^{2}(\Omega)$ and $\mathbf{V}_{h_{\sigma}} \subset \mathbf{W}$ be finite element spaces defined on the partitions $\tau_{h_{u}}$ and $\tau_{h_{\sigma}}$.

Now the semidiscrete positive definite expanded mixed finite element method for (2.5) consists in determining $\left(u_{h}, \lambda_{h}, \sigma_{h}\right) \in X_{h_{u}} \times \mathbf{V}_{h_{\sigma}} \times \mathbf{V}_{h_{\sigma}}$ such that

$$
\begin{aligned}
& \text { (a) }\left(\lambda_{h t}, \mathbf{w}_{h}\right)+\left(\nabla \cdot \sigma_{h}, \nabla \cdot \mathbf{w}_{h}\right)=-\left(f(x, t), \nabla \cdot \mathbf{w}_{h}\right), \quad \forall \mathbf{w}_{h} \in \mathbf{V}_{h_{\sigma}} \\
& \text { (b) }\left(\sigma_{h}, \mathbf{z}_{h}\right)-\left(a \lambda_{h}, \mathbf{z}_{h}\right)-\left(b \int_{0}^{t} \lambda_{h} d s, \mathbf{z}_{h}\right)=0, \quad \forall \mathbf{z}_{h} \in \mathbf{V}_{h_{\sigma}}, \\
& \text { (c) }\left(u_{h t}, v_{h}\right)-\left(\nabla \cdot \sigma_{h}, v_{h}\right)=\left(f(x, t), v_{h}\right), \quad \forall v_{h} \in X_{h_{u}},
\end{aligned}
$$

with given an initial approximation $\left(u_{h^{\prime}}^{0} \lambda_{h}^{0}, \sigma_{h}^{0}\right) \in X_{h_{u}} \times \mathbf{V}_{h_{\sigma}} \times \mathbf{V}_{h_{\sigma}}$.

Remark 2.1. Compared to expanded mixed weak formulation (2.3), the new expanded mixed element system (2.6) is symmetric positive definite, that is to say the gradient function and the flux function system $(2.6)(a, b)$ is symmetric positive definite. And both the gradient equation and the flux equation are separated from its scalar unknown equation (2.6)(c).

Theorem 2.2. There exists a unique discrete solution to the system (2.6).

Proof. Let $\left\{\psi_{i}(x)\right\}_{i=1}^{N_{1}}$ and $\left\{\varphi_{j}(x)\right\}_{j=1}^{N_{2}}$ be bases of $X_{h_{u}}$ and $\mathbf{V}_{h_{\sigma}}$, respectively. Then, we have the following expressions:

$$
u_{h}=\sum_{i=1}^{N_{1}} u_{i}(t) \varphi_{i}(x), \quad \lambda_{h}=\sum_{j=1}^{N_{2}} \lambda_{j}(t) \varphi_{j}(x), \quad \sigma_{h}=\sum_{j=1}^{N_{2}} \sigma_{j}(t) \varphi_{j}(x)
$$


Substituting these expressions into (2.6) and choosing $v_{h}=\psi_{m}, \mathbf{w}_{h}=\mathbf{z}_{h}=\varphi_{l}$, then the problems (2.6) can be written in vector matrix form as: find $\{U(t), \Lambda(t), \Sigma(t)\}$ such that, for all $t \in(0, T]$
(a) $A \Lambda^{\prime}(t)+B \Sigma(t)=F(t)$,
(b) $A \Sigma(t)-C \Lambda(t)-H \int_{0}^{t} \Lambda(s) d s=\mathbf{0}$,
(c) $D U^{\prime}(t)-E \Sigma(t)=G(t)$,

where

$$
\begin{gathered}
A=\left(\left(\varphi_{j}, \varphi_{l}\right)\right)_{N_{2} \times N_{2}{ }^{\prime}} \quad B=\left(\left(\nabla \cdot \varphi_{j}, \nabla \cdot \varphi_{l}\right)\right)_{N_{2} \times N_{2}{ }^{\prime}} \\
C=\left(\left(a \varphi_{j}, \varphi_{l}\right)\right)_{N_{2} \times N_{2}}, \quad D=\left(\left(\psi_{i}, \psi_{m}\right)\right)_{N_{1} \times N_{1}}, \quad H=\left(\left(b \varphi_{j}, \varphi_{l}\right)\right)_{N_{2} \times N_{2}{ }^{\prime}}, \\
E=\left(\left(\nabla \cdot \varphi_{j}, \psi_{m}\right)\right)_{N_{1} \times N_{2}{ }^{\prime}} \quad U(t)=\left(u_{1}(t), u_{2}(t), \ldots, u_{N_{1}}(t)\right)^{T}, \\
\Lambda(t)=\left(\lambda_{1}(t), \lambda_{2}(t), \ldots, \lambda_{N_{2}}(t)\right)^{T}, \quad \Sigma(t)=\left(\sigma_{1}(t), \sigma_{2}(t), \ldots, \sigma_{N_{2}}(t)\right)^{T}, \\
F(t)=\left(\left(-f(t), \nabla \cdot \varphi_{l}\right)\right)_{1 \times N_{2}{ }^{\prime}}^{T} \quad G(t)=\left(\left(f(t), \psi_{m}\right)\right)_{1 \times N_{1}}^{T} .
\end{gathered}
$$

It is easy to see that both $A$ and $D$ are symmetric positive definite. From (2.8), the problems can be written as follows:

$$
\begin{aligned}
& \text { (a) } \Lambda^{\prime}(t)=-A^{-1} B A^{-1}\left(C \Lambda(t)+H \int_{0}^{t} \Lambda(s) d s\right)+A^{-1} F(t), \\
& \text { (b) } \Sigma(t)=A^{-1} C \Lambda(t)+A^{-1} H \int_{0}^{t} \Lambda(s) d s, \\
& \text { (c) } U^{\prime}(t)=D^{-1} E \Sigma(t)+D^{-1} G(t) .
\end{aligned}
$$

Thus, by the theory of differential equations [38, 39], (2.10) has a unique solution, and equivalently (2.6) has a unique solution.

Remark 2.3. It is easy to see that the coefficient matrixes $A, B, C$, and $H$ of system (2.8) are symmetric positive definite. In view of this, the new expanded mixed element system (2.6) is symmetric positive definite. 


\section{Semidiscrete Error Estimates}

Let $X_{h_{u}}$ and $\mathbf{V}_{h_{\sigma}}$ be finite dimensional subspaces of $L^{2}(\Omega)$ and $\mathbf{W}$, respectively, with the inverse property (see [36]) and the following approximation properties (see [40-44]): for $0 \leq$ $p \leq+\infty$ and $r, r^{*}, k$ positive integers

$$
\begin{gathered}
\inf _{\mathbf{w}_{h} \in \mathbf{V}_{h_{\sigma}}}\left\|\mathbf{w}-\mathbf{w}_{h}\right\|_{L^{p}(\Omega)} \leq C h_{\sigma}^{r+1}\|\mathbf{w}\|_{\mathbf{W}^{r+1, p}(\Omega)}, \quad \forall \mathbf{w} \in H(\operatorname{div} ; \Omega) \cap\left[W^{r+1, p}(\Omega)\right]^{d}, \\
\inf _{\mathbf{w}_{h} \in \mathbf{V}_{h_{\sigma}}}\left\|\nabla \cdot\left(\mathbf{w}-\mathbf{w}_{h}\right)\right\|_{L^{p}(\Omega)} \leq C h_{\sigma}^{r^{*}}\|\nabla \cdot \mathbf{w}\|_{W^{r^{*}, p}(\Omega)}, \quad \forall \mathbf{w} \in H(\operatorname{div} ; \Omega) \cap\left[W^{r+1, p}(\Omega)\right]^{d}, \\
\inf _{v_{h} \in X_{h_{u}}}\left\|v-v_{h}\right\|_{L^{p}(\Omega)} \leq C h_{u}^{k+1}\|v\|_{W^{k+1, p}(\Omega)}, \quad \forall v \in L^{2}(\Omega) \cap W^{k+1, p}(\Omega),
\end{gathered}
$$

where $r^{*}=r+1$ for the Brezzi-Douglas-Fortin-Marini spaces [43] and the Raviart-Thomas spaces [42] and $r^{*}=r$ for the Brezzi-Douglas-Marini spaces [40, 43].

For our subsequent error analysis, we introduce two operators. It is well known that, in any one of the classical mixed finite element spaces, there exists an operator $R_{h}$ from $H(\operatorname{div} ; \Omega)$ onto $\mathbf{V}_{h_{\sigma}}$, see $[40-44]$, such that, for $1 \leq p \leq+\infty$,

$$
\begin{gathered}
\left(\nabla \cdot\left(\sigma-R_{h} \sigma\right), \phi_{h}\right)=0, \quad \forall \phi_{h} \in \nabla \cdot \mathbf{V}_{h_{\sigma}}=\left\{\phi_{h}=\nabla \cdot \mathbf{w}_{h}, \mathbf{w}_{h} \in \mathbf{V}_{h_{\sigma}}\right\} ; \\
\left\|\sigma-R_{h} \sigma\right\|_{L^{p}(\Omega)} \leq C h_{\sigma}^{r+1}\|\sigma\|_{\mathbf{W}^{r+1, p}(\Omega)} ; \\
\left\|\nabla \cdot\left(\sigma-R_{h} \sigma\right)\right\|_{L^{p}(\Omega)} \leq C h_{\sigma}^{r^{*}}\|\nabla \cdot \sigma\|_{W^{r^{*}, p}(\Omega)} .
\end{gathered}
$$

We also define the $L^{2}$-project operator $P_{h}$ from $L^{2}(\Omega)$ onto $X_{h_{u}}$ such that

$$
\begin{gathered}
\left(v-P_{h} v, v_{h}\right)=0, \quad \forall v \in L^{2}(\Omega), v_{h} \in X_{h_{u}} ; \\
\left\|v-P_{h} v\right\|_{L^{p}(\Omega)} \leq C h_{u}^{k+1}\|v\|_{H^{k+1, p}(\Omega)}, \quad \forall v \in H^{k+1}(\Omega) .
\end{gathered}
$$

Using the definitions of the operators $R_{h}$ and $P_{h}$, we can easily obtain the following lemma.

Lemma 3.1. Assume that the solution of system (2.5) has the regular properties that $u_{t} \in$ $L^{2}\left(H^{k+1}(\Omega)\right), \quad \lambda_{t}, \lambda_{t t}, \sigma_{t} \in L^{2}\left(\mathbf{H}^{r+1}(\Omega)\right)$, then one has the following estimates:

$$
\begin{aligned}
\left\|\left(\lambda-R_{h} \lambda\right)_{t}\right\|_{\mathbf{L}^{p}(\Omega)} & \leq C h_{\sigma}^{r+1}\left\|\lambda_{t}\right\|_{\mathbf{W}^{r+1, p}(\Omega)}, \\
\left\|\left(\lambda-R_{h} \mathcal{\lambda}\right)_{t t}\right\|_{\mathbf{L}^{p}(\Omega)} & \leq C h_{\sigma}^{r+1}\left\|\lambda_{t t}\right\|_{\mathbf{W}^{r+1, p}(\Omega)}, \\
\left\|\left(\sigma-R_{h} \sigma\right)_{t}\right\|_{\mathbf{L}^{p}(\Omega)} & \leq C h_{\sigma}^{r+1}\left\|\sigma_{t}\right\|_{\mathbf{W}^{r+1, p}(\Omega)} \\
\left\|\left(u-P_{h} u\right)_{t}\right\|_{L^{p}(\Omega)} & \leq C h_{u}^{k+1}\left\|u_{t}\right\|_{H^{k+1, p}(\Omega)} .
\end{aligned}
$$


Journal of Applied Mathematics

Let

$$
\begin{aligned}
& u-u_{h}=u-P_{h} u+P_{h} u-u_{h}=\eta+\zeta, \\
& \lambda-\lambda_{h}=\lambda-R_{h} \lambda+R_{h} \mathcal{\lambda}-\lambda_{h}=\rho+\xi, \\
& \sigma-\sigma_{h}=\sigma-R_{h} \sigma+R_{h} \sigma-\sigma_{h}=\gamma+\theta .
\end{aligned}
$$

Subtracting (2.6) from (2.5) and using projections (3.2) and (3.3), one obtains

$$
\begin{aligned}
& \left(\xi_{t}, \mathbf{w}_{h}\right)+\left(\nabla \cdot \theta, \nabla \cdot \mathbf{w}_{h}\right)=-\left(\rho_{t}, \mathbf{w}_{h}\right), \quad \forall \mathbf{w}_{h} \in \mathbf{V}_{h_{\sigma}} \\
& \left(\theta, \mathbf{z}_{h}\right)-\left(a \xi, \mathbf{z}_{h}\right)-\left(b \int_{0}^{t} \xi d s, \mathbf{z}_{h}\right)=-\left(\gamma, \mathbf{z}_{h}\right)+\left(a \rho, \mathbf{z}_{h}\right)+\left(b \int_{0}^{t} \rho d s, \mathbf{z}_{h}\right), \quad \forall \mathbf{z}_{h} \in \mathbf{V}_{h_{\sigma}}, \\
& \left(\zeta_{t}, v_{h}\right)-\left(\nabla \cdot\left(\sigma-\sigma_{h}\right), v_{h}\right)=0, \quad \forall v_{h} \in X_{h_{u}} .
\end{aligned}
$$

Theorem 3.2. Assume that the approximate properties (3.1) hold, and the solution of system (2.5) has regular properties that $u_{t}, u_{t t} \in L^{2}\left(H^{k+1}(\Omega)\right), \lambda_{t}, \lambda_{t t}, \sigma_{t}, \sigma_{t t} \in L^{2}\left(H^{r+1}(\Omega)\right)$. Then one has the error estimates

$$
\begin{aligned}
& \left\|\lambda-\lambda_{h}\right\|_{L^{\infty}\left(\mathbf{L}^{2}(\Omega)\right)}+\left\|\sigma-\sigma_{h}\right\|_{L^{\infty}\left(\mathbf{L}^{2}(\Omega)\right)} \leq C h_{\sigma}^{r+1}, \\
& \left\|\nabla \cdot\left(\sigma-\sigma_{h}\right)\right\|_{L^{\infty}\left(L^{2}(\Omega)\right)} \leq C h_{\sigma}^{r^{*}}, \\
& \left\|u-u_{h}\right\|_{L^{\infty}\left(L^{2}(\Omega)\right)} \leq C\left(h_{\sigma}^{r^{*}}+h_{u}^{k+1}\right) .
\end{aligned}
$$

Proof. Choose $\mathbf{w}_{h}=\theta$ in (3.6)(a) and $\mathbf{z}_{h}=-\xi_{t}$ in (3.6)(b), and add the two equations to obtain

$$
\begin{aligned}
\frac{1}{2} \frac{d}{d t} \| & a^{1 / 2} \xi\left\|_{\mathrm{L}^{2}(\Omega)}^{2}+\right\| \nabla \cdot \theta \|_{L^{2}(\Omega)}^{2} \\
= & \frac{1}{2}\left(a_{t} \xi, \xi\right)+\left(\gamma, \xi_{t}\right)-\left(a \rho, \xi_{t}\right)-\left(\rho_{t}, \theta\right)-\left(b \int_{0}^{t} \xi d s, \xi t\right)-\left(b \int_{0}^{t} \rho d s, \xi_{t}\right) \\
= & \frac{1}{2}\left(a_{t} \xi, \xi\right)+\frac{d}{d t}(\gamma, \xi)-\left(\gamma_{t}, \xi\right)-\frac{d}{d t}(a \rho, \xi)+\left(a \rho_{t}+a_{t} \rho, \xi\right) \\
& -\left(\rho_{t}, \theta\right)-\frac{d}{d t}\left(b \int_{0}^{t} \xi d s, \xi\right)+\left(b_{t} \int_{0}^{t} \xi d s, \xi\right)+(b \xi, \xi) \\
& -\frac{d}{d t}\left(b \int_{0}^{t} \rho d s, \xi\right)+\left(b_{t} \int_{0}^{t} \rho d s, \xi\right)+(b \rho, \xi) .
\end{aligned}
$$


Integrate with respect to time from 0 to $t$ and apply the Cauchy-Schwarz's inequality and the Young's inequality to obtain

$$
\begin{aligned}
\left\|a^{1 / 2} \xi\right\|_{\mathrm{L}^{2}(\Omega)}^{2}+2 \int_{0}^{t}\|\nabla \cdot \theta\|_{L^{2}(\Omega)}^{2} d s \\
\leq\left\|a^{1 / 2} \xi(0)\right\|_{\mathrm{L}^{2}(\Omega)}^{2}+\|\rho\|_{\mathrm{L}^{2}(\Omega)}^{2}+\|r\|_{\mathrm{L}^{2}(\Omega)}^{2}+\varepsilon\|\xi\|_{\mathrm{L}^{2}(\Omega)}^{2} \\
\quad+C \int_{0}^{t}\left(\|\rho\|_{\mathrm{L}^{2}(\Omega)}^{2}+\left\|\rho_{s}\right\|_{\mathrm{L}^{2}(\Omega)}^{2}+\left\|\gamma_{s}\right\|_{\mathrm{L}^{2}(\Omega)}^{2}+\|\xi\|_{\mathrm{L}^{2}(\Omega)}^{2}+\|\theta\|_{\mathrm{L}^{2}(\Omega)}^{2}\right) d s .
\end{aligned}
$$

Choose $\mathbf{z}_{h}=\theta$ in (3.6)(b) to get

$$
\|\theta\|_{\mathbf{L}^{2}(\Omega)}^{2} \leq C\left(\left\|a^{1 / 2} \xi\right\|_{\mathrm{L}^{2}(\Omega)}^{2}+\|\gamma\|_{\mathrm{L}^{2}(\Omega)}^{2}+\|\rho\|_{\mathrm{L}^{2}(\Omega)}^{2}+\int_{0}^{t}\left(\|\xi\|_{\mathbf{L}^{2}(\Omega)}^{2}+\|\rho\|_{\mathbf{L}^{2}(\Omega)}^{2}\right) d s\right) .
$$

Combining (3.9) and (3.10), we obtain

$$
\begin{aligned}
\|\xi\|_{\mathrm{L}^{2}(\Omega)}^{2} & +\|\theta\|_{\mathrm{L}^{2}(\Omega)}^{2}+2 \int_{0}^{t}\|\nabla \cdot \theta\|_{L^{2}(\Omega)}^{2} d s \\
\leq & \left\|a^{1 / 2} \xi(0)\right\|_{\mathrm{L}^{2}(\Omega)}^{2}+\|\rho\|_{\mathrm{L}^{2}(\Omega)}^{2}+\|r\|_{\mathrm{L}^{2}(\Omega)}^{2}+\varepsilon\|\xi\|_{\mathrm{L}^{2}(\Omega)}^{2} \\
& +C \int_{0}^{t}\left(\|\rho\|_{\mathrm{L}^{2}(\Omega)}^{2}+\left\|\rho_{s}\right\|_{\mathrm{L}^{2}(\Omega)}^{2}+\left\|r_{s}\right\|_{\mathrm{L}^{2}(\Omega)}^{2}+\|\xi\|_{\mathrm{L}^{2}(\Omega)}^{2}+\|\theta\|_{\mathrm{L}^{2}(\Omega)}^{2}\right) d s .
\end{aligned}
$$

Using the fact that $\xi(0)=0$ and Gronwall's lemma, we obtain

$$
\begin{aligned}
& \|\xi\|_{\mathrm{L}^{2}(\Omega)}^{2}+\|\theta\|_{\mathrm{L}^{2}(\Omega)}^{2}+2 \int_{0}^{t}\|\nabla \cdot \theta\|_{L^{2}(\Omega)}^{2} d s \\
& \quad \leq\|\rho\|_{\mathrm{L}^{2}(\Omega)}^{2}+\|\gamma\|_{\mathrm{L}^{2}(\Omega)}^{2}+C \int_{0}^{t}\left(\|\rho\|_{\mathrm{L}^{2}(\Omega)}^{2}+\left\|\rho_{s}\right\|_{\mathrm{L}^{2}(\Omega)}^{2}+\left\|\gamma_{s}\right\|_{\mathrm{L}^{2}(\Omega)}^{2}\right) d s .
\end{aligned}
$$

Differentiating (3.6)(b) and taking $\mathbf{z}_{h}=\xi_{t}$, we obtain

$$
\begin{aligned}
\left(\theta_{t}, \xi_{t}\right)= & \left\|a \xi_{t}\right\|_{\mathrm{L}^{2}(\Omega)}^{2}+\left(a_{t} \xi_{,} \xi_{t}\right)-\left(\gamma_{t}, \xi_{t}\right)+\left(a_{t} \rho+a \rho_{t}, \xi_{t}\right) \\
& +\left(b_{t} \int_{0}^{t} \xi d s, \xi_{t}\right)+\left(b \xi_{,}, \xi_{t}\right)+\left(b_{t} \int_{0}^{t} \rho d s, \xi_{t}\right)+\left(b \rho, \xi_{t}\right)
\end{aligned}
$$

Choosing $\mathbf{w}_{h}=\theta_{t}$ in (3.6)(a), we obtain

$$
\left(\xi_{t}, \theta_{t}\right)+\frac{1}{2} \frac{d}{d t}\|\nabla \cdot \theta\|_{L^{2}(\Omega)}^{2}=-\frac{d}{d t}\left(\rho_{t}, \theta\right)+\left(\rho_{t t}, \theta\right) .
$$


Add (3.13) and (3.14) to obtain

$$
\begin{aligned}
& \left\|a \xi_{t}\right\|_{\mathbf{L}^{2}(\Omega)}^{2}+\frac{1}{2} \frac{d}{d t}\|\nabla \cdot \theta\|_{L^{2}(\Omega)}^{2}=-\frac{d}{d t}\left(\rho_{t}, \theta\right)+\left(\rho_{t t}, \theta\right)-\left(a_{t} \xi_{,} \xi_{t}\right)+\left(\gamma_{t}, \xi_{t}\right)-\left(a_{t} \rho+a \rho_{t}, \xi_{t}\right) \\
& -\left(b_{t} \int_{0}^{t} \xi d s, \xi_{t}\right)-\left(b \xi_{,} \xi_{t}\right)-\left(b_{t} \int_{0}^{t} \rho d s, \xi_{t}\right)-\left(b \rho, \xi_{t}\right) .
\end{aligned}
$$

Integrate (3.15) with respect to time from 0 to $t$ to obtain

$$
\begin{aligned}
& \int_{0}^{t}\left\|\xi_{s}\right\|_{\mathrm{L}^{2}(\Omega)}^{2} d s+\|\nabla \cdot \theta\|_{L^{2}(\Omega)}^{2} \leq\|\rho\|_{\mathrm{L}^{2}(\Omega)}^{2}+\|r\|_{\mathrm{L}^{2}(\Omega)}^{2}+C\left(\|\xi\|_{\mathrm{L}^{2}(\Omega)}^{2}+\int_{0}^{t}\|\xi\|_{\mathrm{L}^{2}(\Omega)}^{2} d s\right) \\
& \quad+C \int_{0}^{t}\left(\|\rho\|_{\mathrm{L}^{2}(\Omega)}^{2}+\left\|\rho_{s}\right\|_{\mathrm{L}^{2}(\Omega)}^{2}+\left\|\rho_{s s}\right\|_{\mathrm{L}^{2}(\Omega)}^{2}+\left\|r_{s}\right\|_{\mathrm{L}^{2}(\Omega)}^{2}\right) d s .
\end{aligned}
$$

Substitute (3.12) into (3.16) to have

$$
\begin{aligned}
\int_{0}^{t}\left\|\xi_{s}\right\|_{\mathrm{L}^{2}(\Omega)}^{2} d s+\|\nabla \cdot \theta\|_{L^{2}(\Omega)}^{2} & \\
\leq & \|\rho\|_{\mathrm{L}^{2}(\Omega)}^{2}+\|\gamma\|_{\mathrm{L}^{2}(\Omega)}^{2}+C \int_{0}^{t}\left(\|\rho\|_{\mathrm{L}^{2}(\Omega)}^{2}+\|\gamma\|_{\mathrm{L}^{2}(\Omega)}^{2}\right. \\
& \left.\quad\left\|\rho_{s}\right\|_{\mathrm{L}^{2}(\Omega)}^{2}+\left\|\rho_{s s}\right\|_{\mathrm{L}^{2}(\Omega)}^{2}+\left\|\gamma_{s}\right\|_{\mathrm{L}^{2}(\Omega)}^{2}\right) d s
\end{aligned}
$$

Choosing $v_{h}=\varsigma$ in (3.6)(b) and applying Cauchy-Schwarz's inequality, we obtain

$$
\|\|_{L^{2}(\Omega)} \leq\left\|\nabla \cdot\left(\sigma-\sigma_{h}\right)\right\|_{L^{2}(\Omega)}
$$

Using Lemma 3.1, (3.17), and Gronwall's lemma, we get

$$
\|\|_{L^{\infty}\left(L^{2}(\Omega)\right)} \leq C\left(h_{\sigma}^{r^{*}}+h_{u}^{k+1}\right)
$$

Using (3.12), (3.17), (3.19), (3.2), (3.3), and Lemma 3.1, we apply the triangle inequality to complete the proof.

\section{Fully Discrete Error Estimates}

In this section, we get the error estimates of fully discrete schemes. For the backward Euler procedure, let $0=t_{0}<t_{1}<t_{2}<\cdots<t_{M}=T$ be a given partition of the time interval $[0, T]$ with step length $\delta=T / M$, for some positive integer $M$. For a smooth function $\phi$ on $[0, T]$, define 
$\phi^{n}=\phi\left(t_{n}\right)$ and $\partial_{t} \phi^{n}=\left(\phi^{n}-\phi^{n-1}\right) / \delta$. For approximating the integrals, we use the composite left rectangle rule

$$
\delta \sum_{j=0}^{n-1} \phi^{j} \approx \int_{0}^{t_{n}} \phi(s) d s
$$

Note that $\phi \in C^{1}[0, T]$, the quadrature error satisfies

$$
\left|\delta \sum_{j=0}^{n-1} \phi^{j}-\int_{0}^{t_{n}} \phi(s) d s\right| \leq C \delta \int_{0}^{t_{n}}\left|\phi_{s}(s)\right| d s .
$$

Equation (2.5) has the following equivalent formulation:

(a) $\left(\partial_{t} \lambda^{n}, \mathbf{w}\right)+\left(\nabla \cdot \sigma^{n}, \nabla \cdot \mathbf{w}\right)=\left(-f^{n}, \nabla \cdot \mathbf{w}\right)+\left(R_{1}^{n}, \mathbf{w}\right), \quad \forall \mathbf{w} \in \mathbf{W}$,

(b) $\left(\sigma^{n}, \mathbf{z}\right)-\left(a^{n} \lambda^{n}, \mathbf{z}\right)-\left(b^{n} \delta \sum_{j=0}^{n-1} \lambda^{j}, \mathbf{z}\right)=-\left(R_{3}^{n}, \mathbf{z}\right), \quad \forall \mathbf{z} \in \mathbf{W}$,

(c) $\left(\partial_{t} u^{n}, v\right)-\left(\nabla \cdot \sigma^{n}, v\right)=\left(f^{n}, v\right)+\left(R_{2}^{n}, v\right), \quad \forall v \in L^{2}(\Omega)$,

where

$$
\begin{aligned}
& R_{1}^{n}=\partial_{t} \lambda^{n}-\lambda_{t}=O\left(\delta \frac{\partial^{2} \lambda}{\partial t^{2}}\right), \quad R_{2}^{n}=\partial_{t} u^{n}-u_{t}=O\left(\delta \frac{\partial^{2} u}{\partial t^{2}}\right), \\
& R_{3}^{n}=\delta \sum_{j=0}^{n-1} \lambda^{j}-\int_{0}^{t_{n}} \lambda(s) d s=O\left(\delta \frac{\partial \lambda}{\partial t}\right) .
\end{aligned}
$$

Now we can formulate a fully discrete scheme based on (4.3).

Fully discrete scheme: find $\left(u_{h^{\prime}}^{n}, \lambda_{h^{\prime}}^{n}, \sigma_{h}^{n}\right) \in X_{h_{u}} \times \mathbf{V}_{h_{\sigma}} \times \mathbf{V}_{h_{\sigma}},(n=1,2, \ldots, M-1)$ such that

(a) $\left(\partial_{t} \lambda_{h^{\prime}}^{n}, \mathbf{w}_{h}\right)+\left(\nabla \cdot \sigma_{h}^{n}, \nabla \cdot \mathbf{w}_{h}\right)=\left(-f^{n}, \nabla \cdot \mathbf{w}_{h}\right), \quad \forall \mathbf{w}_{h} \in \mathbf{V}_{h_{\sigma}}$,

(b) $\left(\sigma_{h}^{n}, \mathbf{z}_{h}\right)-\left(a^{n} \lambda_{h}^{n}, \mathbf{z}_{h}\right)-\left(b^{n} \delta \sum_{j=0}^{n-1} \lambda_{h}^{j}, \mathbf{z}_{h}\right)=0, \quad \forall \mathbf{z}_{h} \in \mathbf{V}_{h_{\sigma}}$,

(c) $\left(\partial_{t} u_{h}^{n}, v_{h}\right)-\left(\nabla \cdot \sigma_{h}^{n}, v_{h}\right)=\left(f^{n}, v_{h}\right), \quad \forall v_{h} \in X_{h_{u}}$

with given an initial approximation $\left(u_{h^{\prime}}^{0} \lambda_{h^{\prime}}^{0} \sigma_{h}^{0}\right) \in X_{h_{u}} \times \mathbf{V}_{h_{\sigma}} \times \mathbf{V}_{h_{\sigma}}$. 
Journal of Applied Mathematics

For fully discrete error estimates, we now split the errors

$$
\begin{aligned}
& u^{n}-u_{h}^{n}=u^{n}-P_{h} u^{n}+P_{h} u^{n}-u_{h}^{n}=\eta^{n}+\varsigma^{n}, \\
& \lambda^{n}-\lambda_{h}^{n}=\lambda^{n}-R_{h} \lambda^{n}+R_{h} \lambda^{n}-\lambda_{h}^{n}=\rho^{n}+\xi^{n}, \\
& \sigma^{n}-\sigma_{h}^{n}=\sigma^{n}-R_{h} \sigma^{n}+R_{h} \sigma^{n}-\sigma_{h}^{n}=\gamma^{n}+\theta^{n} .
\end{aligned}
$$

From (4.3) to (4.5), we then obtain

(a) $\left(\partial_{t} \xi^{n}, \mathbf{w}_{h}\right)+\left(\nabla \cdot \theta^{n}, \nabla \cdot \mathbf{w}_{h}\right)=-\left(\partial_{t} \rho^{n}, \mathbf{w}_{h}\right)+\left(R_{1}^{n}, \mathbf{w}_{h}\right), \quad \forall \mathbf{w}_{h} \in \mathbf{V}_{h_{\sigma}}$

(b) $\left(\theta^{n}, \mathbf{z}_{h}\right)-\left(a^{n} \xi^{n}, \mathbf{z}_{h}\right)-\left(b^{n} \delta \sum_{j=0}^{n-1} \xi^{j}, \mathbf{z}_{h}\right)$

$$
=-\left(\gamma^{n}, \mathbf{z}_{h}\right)+\left(a^{n} \rho^{n}, \mathbf{z}_{h}\right)+\left(b^{n} \delta \sum_{j=0}^{n-1} \rho^{j}, \mathbf{z}_{h}\right)-\left(R_{3}^{n}, \mathbf{z}_{h}\right), \quad \forall \mathbf{z}_{h} \in \mathbf{V}_{h_{\sigma}},
$$

(c) $\left(\partial_{t} S^{n}, v_{h}\right)-\left(\nabla \cdot\left(\theta^{n}+\gamma^{n}\right), v_{h}\right)=\left(R_{2}^{n}, v_{h}\right), \quad \forall v_{h} \in X_{h_{u}}$.

Lemma 4.1. Assume that the solution of system (2.5) has regular properties that $u_{t} \in L^{2}\left(H^{k+1}\right.$ $(\Omega)), \lambda_{t}, \sigma_{t} \in L^{2}\left(\mathbf{H}^{r+1}(\Omega)\right)$. Then one has the estimates

$$
\begin{gathered}
\max _{0 \leq n \leq M}\left\|\partial_{t}\left(\lambda-R_{h} \lambda\right)^{n}\right\|_{L^{2}(\Omega)}+\max _{0 \leq n \leq M}\left\|\partial_{t}\left(\sigma-R_{h} \sigma\right)^{n}\right\|_{L^{2}(\Omega)} \leq C h_{\sigma}^{r+1}, \\
\max _{0 \leq n \leq M}\left\|\partial_{t}\left(u-P_{h} u\right)^{n}\right\|_{L^{2}(\Omega)} \leq C h_{u}^{k+1} .
\end{gathered}
$$

Theorem 4.2. Assume that $\partial^{2} u / \partial t^{2}, \partial u / \partial t \in L^{2}\left(H^{k+1}(\Omega)\right), \partial \lambda / \partial t, \partial^{2} \lambda / \partial t^{2}, \partial \sigma / \partial t, \partial^{2} \sigma / \partial t^{2} \in$ $L^{2}\left(\mathbf{H}^{r+1}(\Omega)\right), u \in L^{\infty}\left(H^{k+1}(\Omega)\right)$, and $\lambda, \sigma \in L^{\infty}\left(\mathbf{H}^{r+1}(\Omega)\right)$, then there exists a constant $C$ such that

$$
\begin{aligned}
& \max _{0 \leq n \leq M}\left\|\left(\lambda-\lambda_{h}\right)^{n}\right\|_{L^{2}(\Omega)}+\max _{0 \leq n \leq M}\left\|\left(\sigma-\sigma_{h}\right)^{n}\right\|_{L^{2}(\Omega)} \leq C\left(h_{\sigma}^{r+1}+\delta\right), \\
& \max _{0 \leq n \leq M}\left\|\nabla \cdot\left(\sigma-\sigma_{h}\right)^{n}\right\|_{L^{2}(\Omega)} \leq C\left(h_{\sigma}^{r^{*}}+\delta\right), \\
& \max _{0 \leq n \leq M}\left\|\left(u-u_{h}\right)^{n}\right\|_{L^{2}(\Omega)} \leq C\left(h_{u}^{k+1}+h_{\sigma}^{r^{*}}+\delta\right) .
\end{aligned}
$$

Proof. Set $\mathbf{w}_{h}=\theta^{n}$ in (4.7)(a) and $\mathbf{z}_{h}=-\partial_{t} \xi^{n}$ in (4.7)(b) and add the two equations to obtain

$$
\begin{gathered}
\left\|\nabla \cdot \theta^{n}\right\|_{L^{2}(\Omega)}^{2}+\left(a^{n} \partial_{t} \xi^{n}, \xi^{n}\right)=-\left(\partial_{t} \rho^{n}, \theta^{n}\right)+\left(R_{1}^{n}, \theta^{n}\right)+\left(\gamma^{n}, \partial_{t} \xi^{n}\right)-\left(a^{n} \rho^{n}, \partial_{t} \xi^{n}\right) \\
-\left(b^{n} \delta \sum_{j=0}^{n-1} \xi^{j}, \partial_{t} \xi^{n}\right)-\left(b^{n} \delta \sum_{j=0}^{n-1} \rho^{j}, \partial_{t} \xi^{n}\right)+\left(R_{3}^{n}, \partial_{t} \xi^{n}\right) .
\end{gathered}
$$


Note that

$$
\begin{aligned}
\partial_{t}\left\|a^{n} \xi^{n}\right\|_{\mathrm{L}^{2}(\Omega)}^{2} & =\frac{\left(a^{n} \xi^{n}, \xi^{n}\right)-\left(a^{n-1} \xi^{n-1}, \xi^{n-1}\right)}{\delta} \\
& =\frac{\left(a^{n} \xi^{n}, \xi^{n}\right)-\left(a^{n} \xi^{n}, \xi^{n-1}\right)+\left(a^{n} \xi^{n}, \xi^{n-1}\right)-\left(a^{n-1} \xi^{n-1}, \xi^{n-1}\right)}{\delta} \\
& =\left(a^{n} \partial_{t} \xi^{n}, \xi^{n}\right)+\left(a^{n} \partial_{t} \xi^{n}, \xi^{n-1}\right)+\left(\partial_{t} a^{n} \xi^{n-1}, \xi^{n-1}\right) \\
& =2\left(a^{n} \partial_{t} \xi^{n}, \xi^{n}\right)-\frac{\left\|\left(a^{n}\right)^{1 / 2}\left(\xi^{n}-\xi^{n-1}\right)\right\|_{\mathbf{L}^{2}(\Omega)}^{2}}{\delta}+\left(\partial_{t} a^{n} \xi^{n-1}, \xi^{n-1}\right) .
\end{aligned}
$$

So, we get

$$
\left(a^{n} \partial_{t} \xi^{n}, \xi^{n}\right)=\frac{1}{2} \partial_{t}\left\|a^{n} \xi^{n}\right\|_{\mathbf{L}^{2}(\Omega)}^{2}+\frac{\left\|\left(a^{n}\right)^{1 / 2}\left(\xi^{n}-\xi^{n-1}\right)\right\|_{\mathrm{L}^{2}(\Omega)}^{2}}{2 \delta}-\frac{1}{2}\left(\partial_{t} a^{n} \xi^{n-1}, \xi^{n-1}\right)
$$

Note that

$$
\begin{gathered}
\left(\gamma^{n}, \partial_{t} \xi^{n}\right)=\frac{\left(\xi^{n}, \gamma^{n}\right)-\left(\xi^{n-1}, \gamma^{n-1}\right)}{\delta}-\left(\partial_{t} \gamma^{n}, \xi^{n-1}\right) \\
\left(a^{n} \rho^{n}, \partial_{t} \xi^{n}\right)=\frac{\left(\xi^{n}, a^{n} \rho^{n}\right)-\left(\xi^{n-1}, a^{n-1} \rho^{n-1}\right)}{\delta}-\left(\partial_{t} a^{n} \rho^{n}, \xi^{n-1}\right) \\
\left(R_{3}^{n}, \partial_{t} \xi^{n}\right)=\frac{\left(\xi^{n}, R_{3}^{n}\right)-\left(\xi^{n-1}, R_{3}^{n-1}\right)}{\delta}-\left(\partial_{t} R_{3}^{n}, \xi^{n-1}\right) . \\
\left(b^{n} \delta \sum_{j=0}^{n-1} \xi^{j}, \partial_{t} \xi^{n}\right)= \\
-\left(\partial_{t} b^{n} \delta \sum_{j=0}^{n-1} \xi^{j}, \xi^{n-1}\right)-\left(b^{n-1} \xi^{n-1}, \xi^{n-1}\right) \\
\left(b^{n} \delta \sum_{j=0}^{n-1} \rho^{j}, \partial_{t} \xi^{n}\right)= \\
-\left(b^{n} \delta \sum_{j=0}^{n-1} \rho^{j}, \xi^{n}\right)-\left(b^{n-1} \delta \sum_{j=0}^{n-2} \rho^{j}, \xi^{n-1}\right)-\left(b^{n-1} \delta \sum_{j=0}^{n-2} \xi^{j}, \xi^{n-1}\right) \\
\delta \\
-\left(\partial_{t} b^{n} \delta \sum_{j=0}^{n-1} \rho^{j}, \xi^{n-1}\right)-\left(b^{n-1} \rho^{n-1}, \xi^{n-1}\right) .
\end{gathered}
$$


Substitute (4.12)-(4.13) into (4.10) to get

$$
\begin{aligned}
\left\|\nabla \cdot \theta^{n}\right\|_{L^{2}(\Omega)}^{2}+\frac{1}{2} \partial_{t}\left\|a^{n} \xi^{n}\right\|_{L^{2}(\Omega)}^{2}+\frac{\left\|\left(a^{n}\right)^{1 / 2}\left(\xi^{n}-\xi^{n-1}\right)\right\|_{L^{2}(\Omega)}^{2}}{2 \delta} \\
=-\left(\partial_{t} \rho^{n}, \theta^{n}\right)+\left(R_{1}^{n}, \theta^{n}\right)+\frac{1}{2}\left(\partial_{t} a^{n} \xi^{n-1}, \xi^{n-1}\right)+\frac{\left(\xi^{n}, \gamma^{n}\right)-\left(\xi^{n-1}, \gamma^{n-1}\right)}{\delta} \\
-\left(\partial_{t} \gamma^{n}, \xi^{n-1}\right)-\frac{\left(\xi^{n}, a^{n} \rho^{n}\right)-\left(\xi^{n-1}, a^{n-1} \rho^{n-1}\right)}{\delta}+\left(\partial_{t} a^{n} \rho^{n}, \xi^{n-1}\right) \\
-\frac{\left(b^{n} \delta \sum_{j=0}^{n-1}\left(\xi^{j}+\rho^{j}\right), \xi^{n}\right)-\left(b^{n-1} \delta \sum_{j=0}^{n-2}\left(\xi^{j}+\rho^{j}\right), \xi^{n-1}\right)}{\delta} \\
+\left(\partial_{t} b^{n} \delta \sum_{j=0}^{n-1}\left(\xi^{j}+\rho^{j}\right), \xi^{n-1}\right)+\left(b^{n-1}\left(\xi^{n-1}+\rho^{n-1}\right), \xi^{n-1}\right) \\
+\frac{\left(\xi^{n}, R_{3}^{n}\right)-\left(\xi^{n-1}, R_{3}^{n-1}\right)}{\delta}-\left(\partial_{t} R_{3}^{n}, \xi^{n-1}\right) .
\end{aligned}
$$

Summing from 1 to $n$, we find that

$$
\begin{aligned}
& \left\|\left(a^{n}\right)^{1 / 2} \xi^{n}\right\|_{\mathrm{L}^{2}(\Omega)}^{2}+2 \delta \sum_{j=1}^{n}\left\|\nabla \cdot \theta^{j}\right\|_{L^{2}(\Omega)}^{2}+\sum_{j=1}^{n}\left\|\left(a^{n}\right)^{1 / 2}\left(\xi^{n}-\xi^{n-1}\right)\right\|_{\mathrm{L}^{2}(\Omega)}^{2} \\
& \leq\left\|\left(a^{0}\right)^{1 / 2} \xi^{0}\right\|_{\mathrm{L}^{2}(\Omega)}^{2}-\left(\xi^{0}, r^{0}\right)+\left(\xi^{0}, a^{0} \rho^{0}\right)+\varepsilon \xi^{n}\left\|_{\mathrm{L}^{2}(\Omega)}^{2}+C\right\| \rho^{n}\left\|_{\mathrm{L}^{2}(\Omega)}^{2}+C\right\| R_{3}^{n} \|_{\mathrm{L}^{2}(\Omega)}^{2} \\
& \quad+C\left\|\gamma^{n}\right\|_{\mathrm{L}^{2}(\Omega)}^{2}+C \delta \sum_{j=1}^{n-1}\left\|\xi^{j}\right\|_{\mathrm{L}^{2}(\Omega)}^{2}+C \delta \sum_{j=1}^{n}\left\|\theta^{j}\right\|_{\mathrm{L}^{2}(\Omega)}^{2} \\
& \quad+C \delta \sum_{j=1}^{n}\left[\left\|\partial_{t} \rho^{j}\right\|_{\mathrm{L}^{2}(\Omega)}^{2}+\left\|\partial_{t} \gamma^{j}\right\|_{\mathrm{L}^{2}(\Omega)}^{2}+\left\|\partial_{t} a^{j} \rho^{j}\right\|_{\mathrm{L}^{2}(\Omega)}^{2}+\left\|\rho^{j}\right\|_{\mathrm{L}^{2}(\Omega)}^{2}+\left\|R_{1}^{j}\right\|_{\mathrm{L}^{2}(\Omega)}^{2}+\left\|\partial_{t} R_{3}^{j}\right\|_{\mathrm{L}^{2}(\Omega)}^{2}\right] .
\end{aligned}
$$

Choose $\mathbf{z}_{h}=\theta^{n}$ in (3.6)(b) to get

$$
\left\|\theta^{n}\right\|_{\mathbf{L}^{2}(\Omega)}^{2} \leq C\left(\left\|\left(a^{n}\right)^{1 / 2} \xi^{n}\right\|_{\mathbf{L}^{2}(\Omega)}^{2}+\left\|r^{n}\right\|_{\mathbf{L}^{2}(\Omega)}^{2}+\left\|\rho^{n}\right\|_{\mathbf{L}^{2}(\Omega)}^{2}\right)+C \delta \sum_{j=1}^{n-1}\left(\left\|\xi^{j}\right\|_{\mathbf{L}^{2}(\Omega)}^{2}+\left\|\rho^{j}\right\|_{\mathbf{L}^{2}(\Omega)}^{2}\right) .
$$


Substitute (4.16) into (4.15) and note that $\xi^{0}=0$ to get

$$
\begin{aligned}
& \left\|\xi^{n}\right\|_{\mathbf{L}^{2}(\Omega)}^{2}+\left\|\theta^{n}\right\|_{\mathbf{L}^{2}(\Omega)}^{2}+2 \delta \sum_{j=1}^{n}\left\|\nabla \cdot \theta^{j}\right\|_{L^{2}(\Omega)}^{2} \\
& \leq C\left\|\rho^{n}\right\|_{\mathrm{L}^{2}(\Omega)}^{2}+C\left\|\gamma^{n}\right\|_{\mathrm{L}^{2}(\Omega)}^{2}+C\left\|R_{3}^{n}\right\|_{\mathrm{L}^{2}(\Omega)}^{2} \\
& +C \delta \sum_{j=1}^{n-1}\left\|\xi^{j}\right\|_{\mathrm{L}^{2}(\Omega)}^{2}+C \delta \sum_{j=1}^{n}\left\|\theta^{j}\right\|_{\mathrm{L}^{2}(\Omega)}^{2} \\
& +C \delta \sum_{j=1}^{n}\left[\left\|\partial_{t} \rho^{j}\right\|_{\mathrm{L}^{2}(\Omega)}^{2}+\left\|\partial_{t} \gamma^{j}\right\|_{\mathrm{L}^{2}(\Omega)}^{2}+\left\|\partial_{t} a^{j} \rho^{j}\right\|_{\mathrm{L}^{2}(\Omega)}^{2}\right. \\
& \left.+\left\|\rho^{j}\right\|_{\mathrm{L}^{2}(\Omega)}^{2}+\left\|R_{1}^{j}\right\|_{\mathrm{L}^{2}(\Omega)}^{2}+\left\|\partial_{t} R_{3}^{j}\right\|_{\mathrm{L}^{2}(\Omega)}^{2}\right] .
\end{aligned}
$$

Using Gronwall's lemma, we obtain

$$
\begin{aligned}
& \left\|\xi^{n}\right\|_{\mathbf{L}^{2}(\Omega)}^{2}+(1-C \delta)\left\|\theta^{n}\right\|_{\mathbf{L}^{2}(\Omega)}^{2}+2 \delta \sum_{j=1}^{n}\left\|\nabla \cdot \theta^{j}\right\|_{\mathbf{L}^{2}(\Omega)}^{2} \\
& \leq C\left\|\rho^{n}\right\|_{\mathbf{L}^{2}(\Omega)}^{2}+C\left\|\gamma^{n}\right\|_{\mathbf{L}^{2}(\Omega)}^{2}+C\left\|R_{3}^{n}\right\|_{\mathbf{L}^{2}(\Omega)}^{2} \\
& +C \delta \sum_{j=1}^{n}\left[\left\|\partial_{t} \rho^{j}\right\|_{L^{2}(\Omega)}^{2}+\left\|\partial_{t} \gamma^{j}\right\|_{\mathbf{L}^{2}(\Omega)}^{2}\right. \\
& \left.\quad+\left\|\partial_{t} a^{j} \rho^{j}\right\|_{\mathbf{L}^{2}(\Omega)}^{2}+\left\|\rho^{j}\right\|_{\mathbf{L}^{2}(\Omega)}^{2}+\left\|R_{1}^{j}\right\|_{\mathbf{L}^{2}(\Omega)}^{2}+\left\|\partial_{t} R_{3}^{j}\right\|_{\mathbf{L}^{2}(\Omega)}^{2}\right] .
\end{aligned}
$$

Note that
(a) $\delta \sum_{j=1}^{n}\left\|R_{1}^{j}\right\|_{\mathrm{L}^{2}(\Omega)}^{2} \leq C \delta^{3} \sum_{j=1}^{n}\left\|\frac{\partial^{2} \lambda}{\partial t^{2}}\right\|_{L^{2}\left(\mathrm{~L}^{2}(\Omega)\right)}^{2} \leq C \delta^{2}\left\|\frac{\partial^{2} \lambda}{\partial t^{2}}\right\|_{L^{\infty}\left(\mathrm{L}^{2}(\Omega)\right)}^{2}$,
(b) $\delta \sum_{j=1}^{n}\left\|\partial_{t} \rho^{j}\right\|_{\mathrm{L}^{2}(\Omega)}^{2} \leq C \delta \sum_{j=1}^{n}\left\|\frac{\partial \rho^{j}}{\partial t}\right\|_{\mathrm{L}^{2}(\Omega)}^{2} \leq C \frac{T}{M} \cdot n h_{\sigma}^{2 r+2} \leq C h_{\sigma}^{2 r+2}$,
(c) $\delta \sum_{j=1}^{n}\left\|\partial_{t} \gamma^{j}\right\|_{L^{2}(\Omega)}^{2} \leq C \delta \sum_{j=1}^{n}\left\|\frac{\partial \gamma^{j}}{\partial t}\right\|_{L^{2}(\Omega)}^{2} \leq C \frac{T}{M} \cdot n h_{\sigma}^{2 r+2} \leq C h_{\sigma}^{2 r+2}$,
(d) $\delta \sum_{j=1}^{n}\left\|\partial_{t} R_{3}^{j}\right\|_{L^{2}(\Omega)}^{2}=C \delta \sum_{j=1}^{n}\left\|\frac{\int_{t_{j-1}}^{t_{j}}\left(\lambda^{j-1}-\lambda\right) d s}{\delta}\right\|_{\mathbf{L}^{2}(\Omega)}^{2} \leq C \delta^{2}\left\|\frac{\partial \lambda}{\partial t}\right\|_{L^{\infty}\left(\mathbf{L}^{2}(\Omega)\right)}^{2}$. 
Journal of Applied Mathematics

Therefore, substituting the above estimates into (4.18) and choosing $\delta_{0}$ in such a way that for $0<\delta \leq \delta_{0},(1-C \delta)>0$, we obtain

$$
\left\|\theta^{n}\right\|_{\mathbf{L}^{2}(\Omega)}^{2}+\left\|\xi^{n}\right\|_{\mathbf{L}^{2}(\Omega)}^{2}+\delta \sum_{j=1}^{n}\left\|\nabla \cdot \theta^{j}\right\|_{L^{2}(\Omega)}^{2} \leq C\left(h_{\sigma}^{2 r+2}+\delta^{2}\right) .
$$

By $(4.7)(b)$, we obtain

$$
\begin{aligned}
\left(\partial_{t} \theta^{n}, \mathbf{z}_{h}\right)= & \left(\frac{a^{n} \xi^{n}-a^{n-1} \xi^{n-1}}{\delta}, \mathbf{z}_{h}\right)-\left(\partial_{t} \gamma^{n}, \mathbf{z}_{h}\right)+\left(\frac{a^{n} \rho^{n}-a^{n-1} \rho^{n-1}}{\delta}, \mathbf{z}_{h}\right) \\
& +\frac{\left(b^{n} \delta \sum_{j=0}^{n-1}\left(\xi^{j}+\rho^{j}\right), \mathbf{z}_{h}\right)-\left(b^{n-1} \delta \sum_{j=0}^{n-2}\left(\xi^{j}+\rho^{j}\right), \mathbf{z}_{h}\right)}{\delta}-\left(\partial_{t} R_{3}^{n}, \mathbf{z}_{h}\right) \\
= & \left(\frac{a^{n} \xi^{n}-a^{n-1} \xi^{n-1}}{\delta}, \mathbf{z}_{h}\right)-\left(\partial_{t} \gamma^{n}, \mathbf{z}_{h}\right)+\left(\frac{a^{n} \rho^{n}-a^{n-1} \rho^{n-1}}{\delta}, \mathbf{z}_{h}\right) \\
& +\left(b^{n}\left(\xi^{n-1}+\rho^{n-1}\right), \mathbf{z}_{h}\right)+\left(\partial_{t} b^{n} \delta \sum_{j=0}^{n-2}\left(\xi^{j}+\rho^{j}\right), \mathbf{z}_{h}\right)-\left(\partial_{t} R_{3}^{n}, \mathbf{z}_{h}\right) .
\end{aligned}
$$

Set $\mathbf{z}_{h}=\partial_{t} \xi^{n}$ in (4.21) to obtain

$$
\begin{aligned}
\left(\partial_{t} \theta^{n}, \partial_{t} \xi^{n}\right)= & \left(\frac{a^{n} \xi^{n}-a^{n-1} \xi^{n-1}}{\delta}, \partial_{t} \xi^{n}\right)-\left(\partial_{t} \gamma^{n}, \partial_{t} \xi^{n}\right)+\left(\frac{a^{n} \rho^{n}-a^{n-1} \rho^{n-1}}{\delta}, \partial_{t} \xi^{n}\right) \\
& +\left(b^{n}\left(\xi^{n-1}+\rho^{n-1}\right), \partial_{t} \xi^{n}\right)+\left(\partial_{t} b^{n} \delta \sum_{j=0}^{n-2}\left(\xi^{j}+\rho^{j}\right), \partial_{t} \xi^{n}\right)-\left(\partial_{t} R_{3}^{n}, \partial_{t} \xi^{n}\right) \\
= & \left\|\left(a^{n}\right)^{1 / 2} \partial_{t} \xi^{n}\right\|_{L^{2}(\Omega)}^{2}+\left(\partial_{t} a^{n} \xi^{n-1}, \partial_{t} \xi^{n}\right)+\left(a^{n} \partial_{t} \rho^{n}, \partial_{t} \xi^{n}\right) \\
& +\left(\partial_{t} a^{n} \rho^{n-1}, \partial_{t} \xi^{n}\right)-\left(\partial_{t} \gamma^{n}, \partial_{t} \xi^{n}\right)+\left(b^{n}\left(\xi^{n-1}+\rho^{n-1}\right), \partial_{t} \xi^{n}\right) \\
& +\left(\partial_{t} b^{n} \delta \sum_{j=0}^{n-2}\left(\xi^{j}+\rho^{j}\right), \partial_{t} \xi^{n}\right)-\left(\partial_{t} R_{3}^{n}, \partial_{t} \xi^{n}\right) .
\end{aligned}
$$

Set $\mathbf{w}_{h}=\partial_{t} \theta^{n}$ in (4.7)(a) to obtain

$$
\left(\partial_{t} \xi^{n}, \partial_{t} \theta^{n}\right)+\frac{1}{2} \partial_{t}\left\|\nabla \cdot \theta^{n}\right\|_{L^{2}(\Omega)}^{2}+\frac{\left\|\nabla \cdot\left(\theta^{n}-\theta^{n-1}\right)\right\|_{L^{2}(\Omega)}^{2}}{2 \delta}=-\left(\partial_{t} \rho^{n}, \partial_{t} \theta^{n}\right)+\left(R_{1}^{n}, \partial_{t} \theta^{n}\right) .
$$


Substitute (4.22) into (4.23) to get

$$
\begin{aligned}
\left\|\left(a^{n}\right)^{1 / 2} \partial_{t} \xi^{n}\right\|_{L^{2}(\Omega)}^{2}+\frac{1}{2} \partial_{t}\left\|\nabla \cdot \theta^{n}\right\|_{L^{2}(\Omega)}^{2}+\frac{\left\|\nabla \cdot\left(\theta^{n}-\theta^{n-1}\right)\right\|_{L^{2}(\Omega)}^{2}}{2 \delta} \\
=-\left(\partial_{t} \rho^{n}, \partial_{t} \theta^{n}\right)-\left(\partial_{t} a^{n} \xi^{n-1}, \partial_{t} \xi^{n}\right)-\left(a^{n} \partial_{t} \rho^{n}, \partial_{t} \xi^{n}\right) \\
-\left(\partial_{t} a^{n} \rho^{n-1}, \partial_{t} \xi^{n}\right)+\left(\partial_{t} \gamma^{n}, \partial_{t} \xi^{n}\right)-\left(b^{n}\left(\xi^{n-1}+\rho^{n-1}\right), \partial_{t} \xi^{n}\right) \\
+\left(R_{1}^{n}, \partial_{t} \theta^{n}\right)-\left(\partial_{t} b^{n} \delta \sum_{j=0}^{n-2}\left(\xi^{j}+\rho^{j}\right), \partial_{t} \xi^{n}\right)+\left(\partial_{t} R_{3}^{n}, \partial_{t} \xi^{n}\right) .
\end{aligned}
$$

Take $\mathbf{z}_{h}=\partial_{t} \theta^{n}$ in (4.21) to obtain

$$
\begin{aligned}
\left\|\partial_{t} \theta^{n}\right\|_{\mathbf{L}^{2}(\Omega)}^{2}= & \left(\frac{a^{n} \xi^{n}-a^{n-1} \xi^{n-1}}{\delta}, \partial_{t} \theta^{n}\right)-\left(\partial_{t} \gamma^{n}, \partial_{t} \theta^{n}\right) \\
& +\left(\frac{a^{n} \rho^{n}-a^{n-1} \rho^{n-1}}{\delta}, \partial_{t} \theta^{n}\right)+\left(b^{n}\left(\xi^{n-1}+\rho^{n-1}\right), \partial_{t} \theta^{n}\right) \\
& +\left(\partial_{t} b^{n} \delta \sum_{j=0}^{n-2}\left(\xi^{j}+\rho^{j}\right), \partial_{t} \theta^{n}\right)-\left(\partial_{t} R_{3}^{n}, \partial_{t} \theta^{n}\right) \\
= & \left(a^{n} \partial_{t} \xi^{n}, \partial_{t} \theta^{n}\right)+\left(\partial_{t} a^{n} \xi^{n-1}, \partial_{t} \theta^{n}\right)-\left(\partial_{t} \gamma^{n}, \partial_{t} \theta^{n}\right)+\left(a^{n} \partial_{t} \rho^{n}, \partial_{t} \theta^{n}\right) \\
& +\left(\partial_{t} a^{n} \rho^{n-1}, \partial_{t} \theta^{n}\right)+\left(b^{n}\left(\xi^{n-1}+\rho^{n-1}\right), \partial_{t} \theta^{n}\right) \\
& +\left(\partial_{t} b^{n} \delta \sum_{j=0}^{n-2}\left(\xi^{j}+\rho^{j}\right), \partial_{t} \theta^{n}\right)-\left(\partial_{t} R_{3}^{n}, \partial_{t} \theta^{n}\right) .
\end{aligned}
$$

Add (4.24) and (4.25) to get

$$
\begin{aligned}
\left\|\left(a^{n}\right)^{1 / 2} \partial_{t} \xi^{n}\right\|_{L^{2}(\Omega)}^{2}+\left\|\partial_{t} \theta^{n}\right\|_{L^{2}(\Omega)}^{2}+\frac{1}{2} \partial_{t}\left\|\nabla \cdot \theta^{n}\right\|_{L^{2}(\Omega)}^{2}+\frac{\left\|\nabla \cdot\left(\theta^{n}-\theta^{n-1}\right)\right\|_{L^{2}(\Omega)}^{2}}{2 \delta} \\
=-\left(\partial_{t} \rho^{n}, \partial_{t} \theta^{n}\right)-\left(\partial_{t} a^{n} \xi^{n-1}, \partial_{t} \xi^{n}\right)-\left(a^{n} \partial_{t} \rho^{n}, \partial_{t} \xi^{n}\right)-\left(\partial_{t} a^{n} \rho^{n-1}, \partial_{t} \xi^{n}\right) \\
\quad+\left(\partial_{t} \gamma^{n}, \partial_{t} \xi^{n}\right)+\left(a^{n} \partial_{t} \xi^{n}, \partial_{t} \theta^{n}\right)+\left(\partial_{t} a^{n} \xi^{n-1}, \partial_{t} \theta^{n}\right)-\left(\partial_{t} \gamma^{n}, \partial_{t} \theta^{n}\right) \\
+\left(a^{n} \partial_{t} \rho^{n}, \partial_{t} \theta^{n}\right)+\left(\partial_{t} a^{n} \rho^{n-1}, \partial_{t} \theta^{n}\right)+\left(R_{1}^{n}, \partial_{t} \theta^{n}\right)-\left(\partial_{t} R_{3}^{n}, \partial_{t} \theta^{n}+\partial_{t} \xi^{n}\right) \\
+\left(b^{n}\left(\xi^{n-1}+\rho^{n-1}\right), \partial_{t} \theta^{n}+\partial_{t} \xi^{n}\right)+\left(\partial_{t} b^{n} \delta \sum_{j=0}^{n-2}\left(\xi^{j}+\rho^{j}\right), \partial_{t} \theta^{n}+\partial_{t} \xi^{n}\right) .
\end{aligned}
$$


Apply Cauchy-Schwarz's inequality and Young's inequality to obtain

$$
\begin{aligned}
& a_{\min }\left\|\partial_{t} \xi^{n}\right\|_{\mathrm{L}^{2}(\Omega)}^{2}+\left\|\partial_{t} \theta^{n}\right\|_{\mathrm{L}^{2}(\Omega)}^{2}+\frac{1}{2} \partial_{t}\left\|\nabla \cdot \theta^{n}\right\|_{L^{2}(\Omega)}^{2} \\
& \leq \varepsilon\left(\left\|\partial_{t} \xi^{n}\right\|_{\mathrm{L}^{2}(\Omega)}^{2}+\left\|\partial_{t} \theta^{n}\right\|_{\mathrm{L}^{2}(\Omega)}^{2}\right) \\
& \quad+C\left[\left\|\xi^{n-1}\right\|_{\mathrm{L}^{2}(\Omega)}^{2}+\left\|\partial_{t} \rho^{n}\right\|_{\mathrm{L}^{2}(\Omega)}^{2}+\left\|\partial_{t} \gamma^{n}\right\|_{\mathrm{L}^{2}(\Omega)}^{2}+\left\|\rho^{n-1}\right\|_{\mathrm{L}^{2}(\Omega)}^{2}+\left\|R_{1}^{n}\right\|_{\mathrm{L}^{2}(\Omega)}^{2}+\left\|\partial_{t} R_{3}^{n}\right\|_{\mathrm{L}^{2}(\Omega)}^{2}\right] \\
& \quad+C \delta^{2} \sum_{j=0}^{n-2}\left(\left\|\xi^{j}\right\|_{\mathrm{L}^{2}(\Omega)}^{2}+\left\|\rho^{j}\right\|_{\mathrm{L}^{2}(\Omega)}^{2}\right) .
\end{aligned}
$$

Using (4.19) and (4.20) and summing from 1 to $n$, we obtain

$$
\left\|\nabla \cdot \theta^{n}\right\|_{L^{2}(\Omega)}^{2}+\delta \sum_{j=1}^{n}\left(\left\|\partial_{t} \xi^{n}\right\|_{L^{2}(\Omega)}^{2}+\left\|\partial_{t} \theta^{n}\right\|_{\mathbf{L}^{2}(\Omega)}^{2}\right) \leq C\left(h_{\sigma}^{2 r+2}+\delta^{2}\right)
$$

Choosing $v_{h}=\varsigma^{n}$ in (4.7)(c) and applying Cauchy-Schwarz's inequality, Young's inequality, and (4.28), we have

$$
\begin{gathered}
\left\|\varsigma^{n}\right\|_{L^{2}(\Omega)}^{2}-\left\|\varsigma^{n-1}\right\|_{L^{2}(\Omega)}^{2}=2 \delta\left(\nabla \cdot\left(\sigma-\sigma_{h}\right)^{n}, \varsigma^{n}\right)+2 \delta\left(R_{2}^{n}, \varsigma^{n}\right) \\
\leq C \delta\left[\left\|\nabla \cdot\left(\sigma-\sigma_{h}\right)^{n}\right\|_{L^{2}(\Omega)}^{2}+\left\|R_{2}^{n}\right\|_{L^{2}(\Omega)}^{2}+\left\|\varsigma^{n}\right\|_{L^{2}(\Omega)}^{2}\right] .
\end{gathered}
$$

Summing from 1 to $n$ and using the Gronwall lemma, we obtain

$$
\left\|S^{n}\right\|_{L^{2}(\Omega)}^{2} \leq\left\|\varsigma^{0}\right\|_{L^{2}(\Omega)}^{2}+C \delta \sum_{j=1}^{n}\left[\left\|\nabla \cdot\left(\sigma-\sigma_{h}\right)^{j}\right\|_{L^{2}(\Omega)}^{2}+\left\|R_{2}^{j}\right\|_{L^{2}(\Omega)}^{2}\right] .
$$

Note that

$$
\delta \sum_{j=1}^{n}\left\|R_{2}^{j}\right\|_{L^{2}(\Omega)}^{2} \leq C \delta^{3} \sum_{j=1}^{n}\left\|\frac{\partial^{2} u}{\partial t^{2}}\right\|_{L^{2}\left(L^{2}(\Omega)\right)}^{2} \leq C \delta^{2}\left\|\frac{\partial^{2} u}{\partial t^{2}}\right\|_{L^{\infty}\left(L^{2}(\Omega)\right)}^{2} .
$$

Substituting (4.31) into (4.30) and using (3.2), (4.20), and the triangle inequality, we get

$$
\left\|S^{n}\right\|_{L^{2}(\Omega)}^{2} \leq C\left(h_{u}^{2 k+2}+h_{\sigma}^{2 r *}+\delta^{2}\right) .
$$

Combining (3.2), (3.3), (4.20), (4.28), (4.32), and Lemma 4.1, we apply the triangle inequality to complete the proof. 
Table 1: The errors and convergence order.

\begin{tabular}{lcccc}
\hline$(h, \delta)$ & $\left\|u-u_{h}\right\|_{L^{\infty}\left(L^{2}(\Omega)\right)}$ & Order & $\left\|\lambda-\lambda_{h}\right\|_{L^{\infty}\left(\mathrm{L}^{2}(\Omega)\right)}$ & Order \\
\hline$\left(\frac{\sqrt{2}}{8}, \frac{1}{8}\right)$ & $1.4527 e-002$ & & $1.3532 e-001$ & \\
$\left(\frac{\sqrt{2}}{16}, \frac{1}{16}\right)$ & $6.5250 e-003$ & 1.15 & $6.9202 e-002$ & 0.97 \\
$\left(\frac{\sqrt{2}}{32}, \frac{1}{32}\right)$ & $3.0841 e-003$ & 1.08 & $3.5363 e-002$ & 0.97 \\
\hline$(h, \delta)$ & $\left\|\sigma-\sigma_{h}\right\|_{L^{\infty}\left(\mathrm{L}^{2}(\Omega)\right)}$ & Order & $\left\|\sigma-\sigma_{h}\right\|_{L^{\infty}(H(\mathrm{div} ; \Omega))}$ & Order \\
\hline$\left(\frac{\sqrt{2}}{8}, \frac{1}{8}\right)$ & $3.3512 e-001$ & & $3.8892 e-001$ & \\
$\left(\frac{\sqrt{2}}{16}, \frac{1}{16}\right)$ & $1.6734 e-001$ & & & \\
$\left(\frac{\sqrt{2}}{32}, \frac{1}{32}\right)$ & $8.3746 e-002$ & 1.00 & $1.7291 e-001$ & 1.17 \\
\hline
\end{tabular}

\section{Numerical Example}

In this section, we analyse some numerical results to illustrate the efficiency of the proposed method. We consider the following 2D parabolic partial integrodifferential equations with initial-boundary value condition:

$$
\begin{gathered}
u_{t}-\nabla \cdot\left(a(x, t) \nabla u+b(x, t) \int_{0}^{t} \nabla u d s\right)=f(x, t), \quad(x, t) \in \Omega \times J, \\
u(x, t)=0, \quad(x, t) \in \partial \Omega \times \bar{J}, \\
u(x, 0)=\sin \left(\pi x_{1}\right) \sin \left(\pi x_{2}\right), \quad x \in \Omega,
\end{gathered}
$$

where $\Omega=[0,1] \times[0,1], J=(0,1], a(x, t)=1+x_{1}^{2}+2 x_{2}^{2}, b(x, t)=1+2 x_{1}^{2}+x_{2}^{2}, x=\left(x_{1}, x_{2}\right)$, and $f(x, t)$ is chosen so that the exact solution for the scalar unknown function is

$$
u(x, t)=e^{-t} \sin \left(\pi x_{1}\right) \sin \left(\pi x_{2}\right) .
$$

The corresponding exact gradient is

$$
\lambda(x, t)=\left(\lambda_{1}, \lambda_{2}\right)=\left(\pi e^{-t} \cos \left(\pi x_{1}\right) \sin \left(\pi x_{2}\right), \pi e^{-t} \sin \left(\pi x_{1}\right) \cos \left(\pi x_{2}\right)\right),
$$




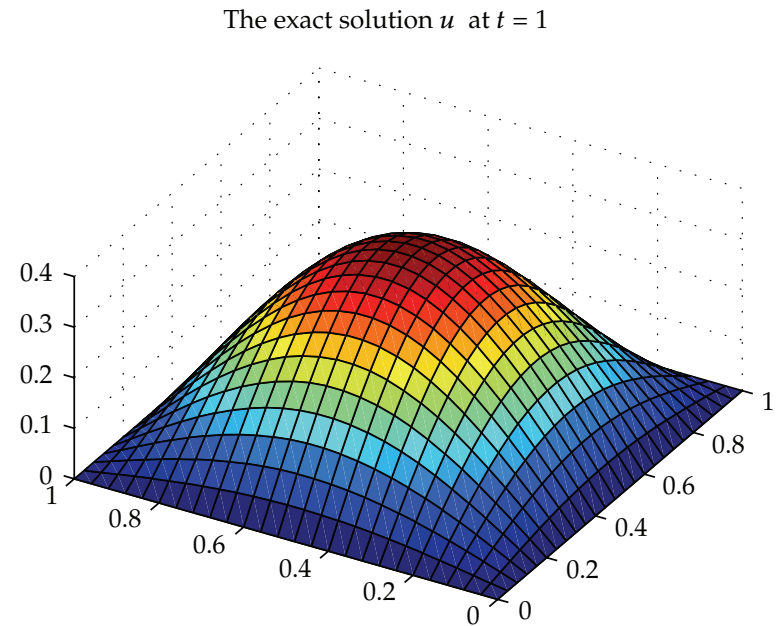

Figure 1: The exact solution $u$.

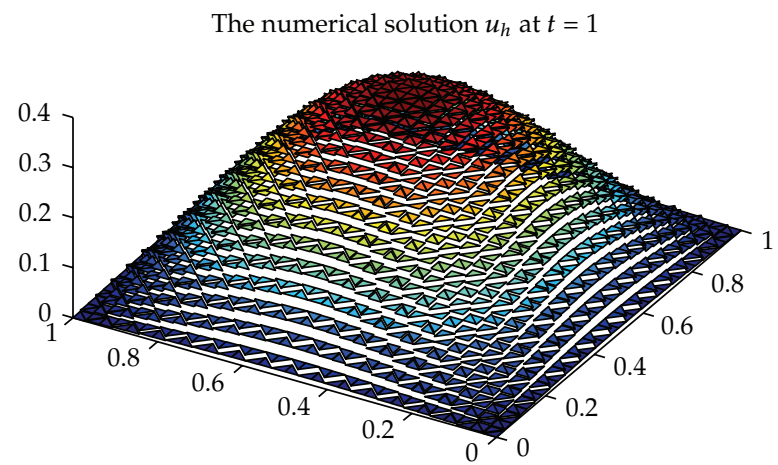

Figure 2: The numerical solution $u_{h}$.

and its exact flux is

$$
\begin{array}{r}
\sigma(x, t)=\left(\sigma_{1}, \sigma_{2}\right)=\left(\pi\left(1+\left(2-e^{-t}\right) x_{1}^{2}+\left(1+e^{-t}\right) x_{2}^{2}\right) \cos \left(\pi x_{1}\right) \sin \left(\pi x_{2}\right),\right. \\
\left.\pi\left(1+\left(2-e^{-t}\right) x_{1}^{2}+\left(1+e^{-t}\right) x_{2}^{2}\right) \sin \left(\pi x_{1}\right) \cos \left(\pi x_{2}\right)\right) .
\end{array}
$$

Dividing the domain $\Omega$ into the triangulations of mesh size $h_{u}=h_{\sigma}=h$ uniformly, considering the piecewise constant space $X_{h_{u}}$ with index $k=0$ for the scalar unknown function $u$ and the lowest-order Raviart-Thomas triangular space $\mathbf{V}_{h_{\sigma}}[42,45]$ for the gradient $\lambda$ and the flux $\sigma$ and using the backward Euler procedure with uniform time step length $\delta=1 / M$, we obtain some convergence results for $\left\|u-u_{h}\right\|_{L^{\infty}\left(L^{2}(\Omega)\right)},\left\|\lambda-\lambda_{h}\right\|_{L^{\infty}\left(\mathbf{L}^{2}(\Omega)\right)}$, $\left\|\sigma-\sigma_{h}\right\|_{L^{\infty}\left(\mathbf{L}^{2}(\Omega)\right)}$ and $\left\|\sigma-\sigma_{h}\right\|_{L^{\infty}(H(\operatorname{div} ; \Omega))}$ with $h=\sqrt{2} \delta=\sqrt{2} / 8, \sqrt{2} / 16, \sqrt{2} / 32$ in Table 1. With time $t=1, h=\sqrt{2} \delta=\sqrt{2} / 32$, the exact solution $u, \lambda, \sigma$ is shown in Figures 1, 3, and 5 , respectively, and the corresponding numerical solution $u_{h}, \lambda_{h}, \sigma_{h}$ is shown in Figures 2,4 , and 6 , respectively. 
The exact solution $\lambda=\left(\lambda_{1}, \lambda_{2}\right)$ at $t=1$

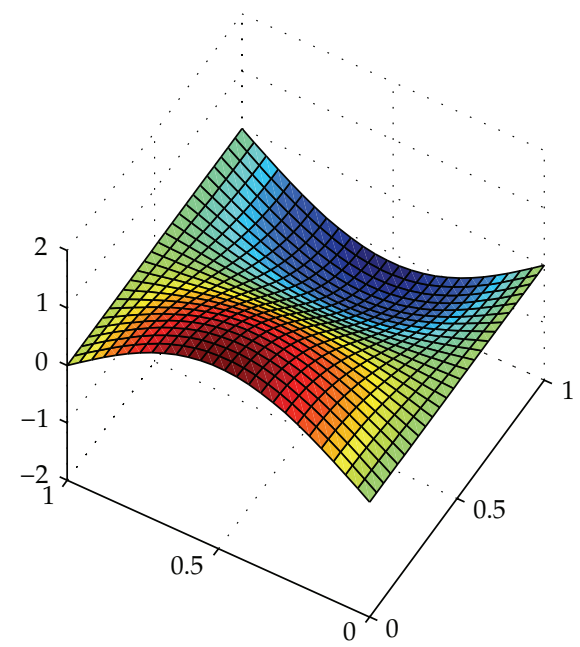

(a)
The exact solution $\lambda=\left(\lambda_{1}, \lambda_{2}\right)$ at $t=1$

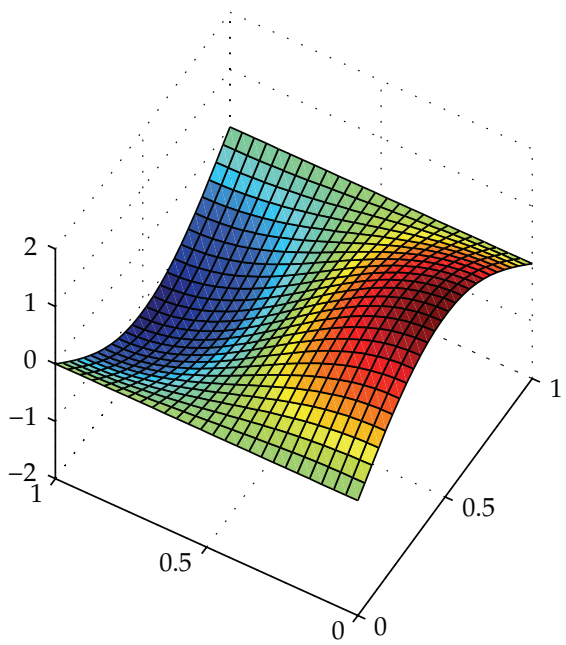

(b)

Figure 3: The exact gradient $\lambda_{1}(\mathrm{a})$ and $\lambda_{2}(\mathrm{~b})$.

The numerical solution $\lambda_{h}=\left(\lambda_{1 h}, \lambda_{2 h}\right)$ at $t=1$

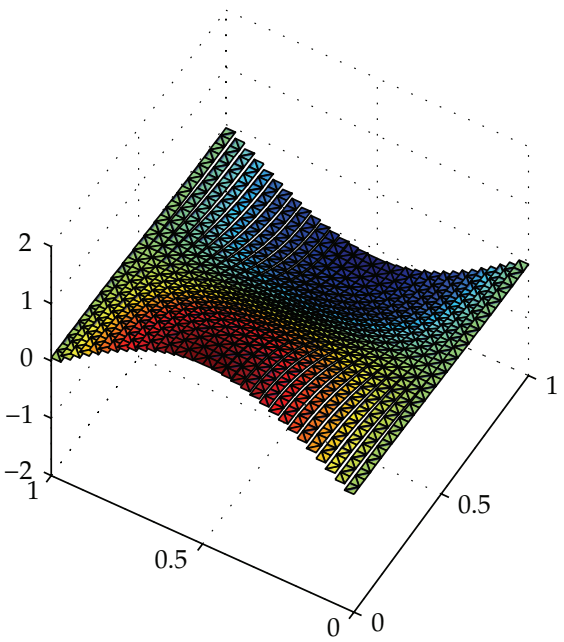

(a)
The numerical solution $\lambda_{h}=\left(\lambda_{1 h}, \lambda_{2 h}\right)$ at $t=1$

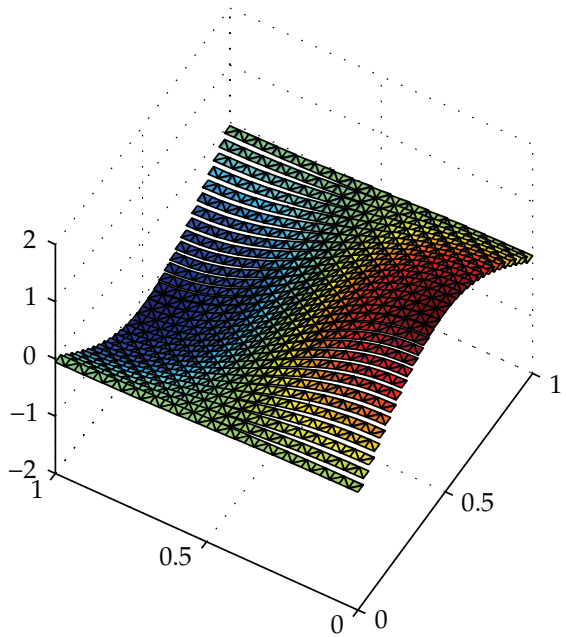

(b)

Figure 4: The numerical gradient $\lambda_{1 h}$ (a) and $\lambda_{2 h}$ (b).

We can see from Table 1 that the convergence rate is order 1 which confirms the theoretical results of Theorem 4.2 for the above chosen spaces $X_{h_{u}}$ and $\mathbf{V}_{h_{\sigma}}$. The numerical results in Table 1 and Figures 1-6 show that new positive definite expanded mixed scheme is efficient. 


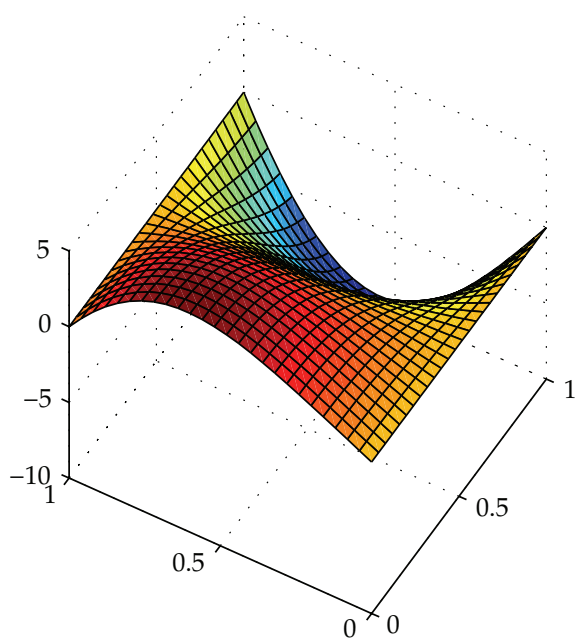

(a)

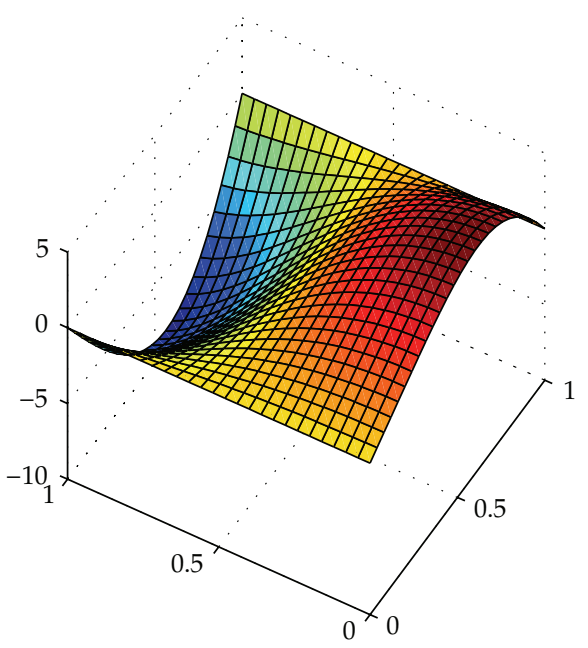

(b)

Figure 5: The exact flux $\sigma_{1}(\mathrm{a})$ and $\sigma_{2}(\mathrm{~b})$.

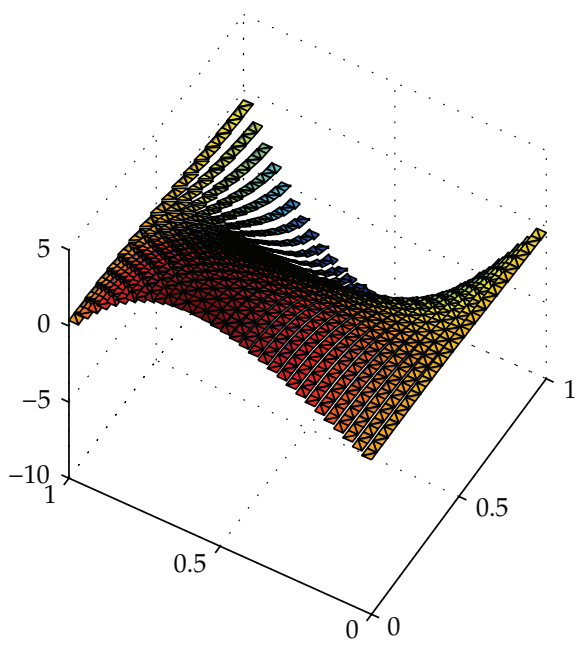

(a)

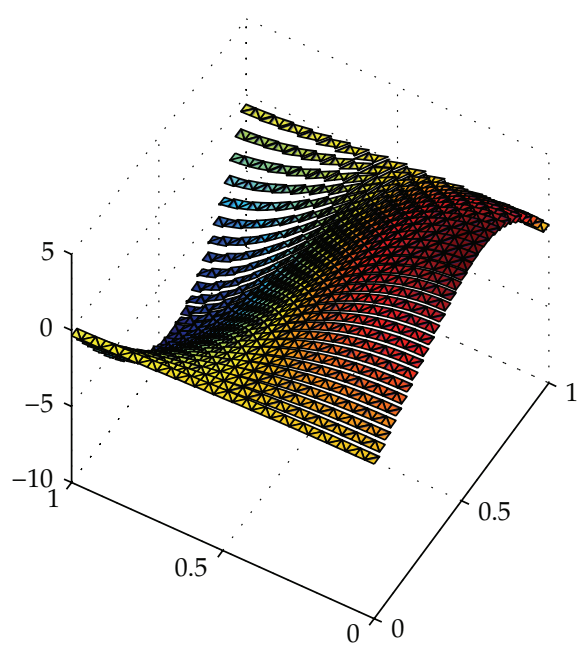

(b)

Figure 6: The numerical flux $\sigma_{1 h}(\mathrm{a})$ and $\sigma_{2 h}(\mathrm{~b})$.

\section{Concluding Remarks}

In the paper, a new expanded mixed finite element method based on a positive definite system is proposed for parabolic partial integrodifferential equation. Compared to expanded mixed method and standard mixed methods, the new expanded mixed element system is symmetric positive definite and both the gradient equation and the flux equation are separated from its scalar unknown equation. The existence and uniqueness for semidiscrete 
scheme are proved and error estimates are derived for both semidiscrete and fully discrete schemes. Finally, some numerical results are provided to confirm our theoretical analysis. In the near further, we will study the others evolution equations such as hyperbolic wave equation, and miscible displacement of compressible flow in porous media.

\section{Acknowledgments}

The authors would like to thank the Editor and the referees for their valuable suggestions and comments. This work is supported by the National Natural Science Fund of China (no. 11061021), the Scientific Research Projection of Higher Schools of Inner Mongolia (no. NJ10006, no. NJ10016, no. NJZZ12011), the Program of Higher-level talents of Inner Mongolia University (SPH-IMU, no. Z200901004, no. 125119), and the YSF of Inner Mongolia University (no. ND0702).

\section{References}

[1] T. Zhang and C. J. Li, "Superconvergence of finite element approximations to parabolic and hyperbolic integro-differential equations," Northeastern Mathematical Journal, vol. 17, no. 3, pp. 279-288, 2001.

[2] A. K. Pani and T. E. Peterson, "Finite element methods with numerical quadrature for parabolic integrodifferential equations," SIAM Journal on Numerical Analysis, vol. 33, no. 3, pp. 1084-1105, 1996.

[3] A. K. Pani and R. K. Sinha, "Error estimates for semidiscrete Galerkin approximation to a time dependent parabolic integro-differential equation with nonsmooth data," Calcolo, vol. 37, no. 4, pp. 181205, 2000.

[4] Y. P. Lin, V. Thomée, and L. B. Wahlbin, "Ritz-Volterra projections to finite-element spaces and applications to integrodifferential and related equations," SIAM Journal on Numerical Analysis, vol. 28, no. 4, pp. 1047-1070, 1991.

[5] T. Zhang, Finite Element Methods for Partial Differentio-Integral Equations, Chinese Science Press, Beijing, China, 2009.

[6] R. K. Sinha, R. E. Ewing, and R. D. Lazarov, "Mixed finite element approximations of parabolic integro-differential equations with nonsmooth initial data," SIAM Journal on Numerical Analysis, vol. 47, no. 5, pp. 3269-3292, 2009.

[7] Z. Jiang, " $L^{\infty}\left(L^{2}\right)$ and $L^{\infty}\left(L^{\infty}\right)$ error estimates for mixed methods for integro-differential equations of parabolic type," Mathematical Modelling and Numerical Analysis, vol. 33, no. 3, pp. 531-546, 1999.

[8] H. Guo and H. X. Rui, "Least-squares Galerkin procedures for parabolic integro-differential equations," Applied Mathematics and Computation, vol. 150, no. 3, pp. 749-762, 2004.

[9] D. Y. Shi and H. H. Wang, "An $H^{1}$-Galerkin nonconforming mixed finite element method for integrodifferential equation of parabolic type," Journal of Mathematical Research and Exposition, vol. 29, no. 5, pp. 871-881, 2009.

[10] H. R. Li and Q. Li, "Finite volume element methods for nonlinear parabolic integro-differential problems," Journal of the Korean Society for Industrial and Applied Mathematics, vol. 7, no. 2, pp. 35-49, 2003.

[11] Z. X. Chen, "Expanded mixed finite element methods for linear second order elliptic problems I," IMA preprint series \# 1219, Institute for Mathematics and Its Applications, University of Minnesota, Minneapolis, Minn, USA, 1994.

[12] Z. X. Chen, "Expanded mixed finite element methods for linear second-order elliptic problems. I," RAIRO Modélisation Mathématique et Analyse Numérique, vol. 32, no. 4, pp. 479-499, 1998.

[13] T. Arbogast, M. F. Wheeler, and I. Yotov, "Mixed finite elements for elliptic problems with tensor coefficients as cell-centered finite differences," SIAM Journal on Numerical Analysis, vol. 34, no. 2, pp. 828-852, 1997.

[14] Z. X. Chen, "Expanded mixed finite element methods for quasilinear second order elliptic problems. II," RAIRO Modélisation Mathématique et Analyse Numérique, vol. 32, no. 4, pp. 501-520, 1998.

[15] Z. X. Chen, "Analysis of expanded mixed methods for fourth-order elliptic problems," Numerical Methods for Partial Differential Equations, vol. 13, no. 5, pp. 483-503, 1997. 
[16] Y. Liu, H. Li, and Z. C. Wen, "Expanded mixed finite element method for a second-order linear parabolic differential equation," Numerical Mathematics. A Journal of Chinese Universities, vol. 30, no. 3, pp. 234-249, 2008.

[17] C. S. Woodward and C. N. Dawson, "Analysis of expanded mixed finite element methods for a nonlinear parabolic equation modeling flow into variably saturated porous media," SIAM Journal on Numerical Analysis, vol. 37, no. 3, pp. 701-724, 2000.

[18] L. Wu and M. B. Allen, "A two-grid method for mixed finite-element solution of reaction-diffusion equations," Numerical Methods for Partial Differential Equations, vol. 15, no. 3, pp. 317-332, 1999.

[19] Y. P. Chen, Y. Q. Huang, and D. H. Yu, "A two-grid method for expanded mixed finite-element solution of semilinear reaction-diffusion equations," International Journal for Numerical Methods in Engineering, vol. 57, no. 2, pp. 193-209, 2003.

[20] Y. P. Chen, H. W. Liu, and S. Liu, "Analysis of two-grid methods for reaction-diffusion equations by expanded mixed finite element methods," International Journal for Numerical Methods in Engineering, vol. 69, no. 2, pp. 408-422, 2007.

[21] Y. P. Chen and L. Li, " $L^{p}$ error estimates of two-grid schemes of expanded mixed finite element methods," Applied Mathematics and Computation, vol. 209, no. 2, pp. 197-205, 2009.

[22] Y. P. Chen, P. Luan, and Z. L. Lu, "Analysis of two-grid methods for nonlinear parabolic equations by expanded mixed finite element methods," Advances in Applied Mathematics and Mechanics, vol. 1, no. 6, pp. 830-844, 2009.

[23] H. L. Song and Y. R. Yuan, "The expanded upwind-mixed multi-step method for the miscible displacement problem in three dimensions," Applied Mathematics and Computation, vol. 195, no. 1, pp. 100-109, 2008.

[24] L. Guo and H. Z. Chen, "An expanded characteristic-mixed finite element method for a convectiondominated transport problem," Journal of Computational Mathematics, vol. 23, no. 5, pp. 479-490, 2005.

[25] H. Z. Chen and H. Wang, "An optimal-order error estimate on an $H^{1}$-Galerkin mixed method for a nonlinear parabolic equation in porous medium flow," Numerical Methods for Partial Differential Equations, vol. 26, no. 1, pp. 188-205, 2010.

[26] Y. Liu and H. Li, “A new mixed finite element method for pseudo-hyperbolic equation," Mathematica Applicata, vol. 23, no. 1, pp. 150-157, 2010.

[27] Y. Liu, Analysis and numerical simulation of nonstandard mixed element methods [Ph.D. thesis], Inner Mongolia University, Hohhot, China, 2011.

[28] Z. W. Jiang and A. Q. Li, "Expanded mixed finite element methods for the problem of purely longitudinal motion of a homogeneous bar," Journal of Computational and Applied Mathematics, vol. 235, no. 8, pp. 2157-2169, 2011.

[29] A. L. Zhu, Z. W. Jiang, and Q. Xu, “Expanded mixed covolume method for a linear integro-differential equation of parabolic type," Numerical Mathematics. A Journal of Chinese Universities, vol. 31, no. 3, pp. 193-205, 2009.

[30] H. X. Rui and T. C. Lu, "An expanded mixed covolume method for elliptic problems," Numerical Methods for Partial Differential Equations, vol. 21, no. 1, pp. 8-23, 2005.

[31] D. Kim and E. J. Park, "A posteriori error estimator for expanded mixed hybrid methods," Numerical Methods for Partial Differential Equations, vol. 23, no. 2, pp. 330-349, 2007.

[32] D. P. Yang, "A splitting positive definite mixed element method for miscible displacement of compressible flow in porous media," Numerical Methods for Partial Differential Equations, vol. 17, no. 3, pp. 229-249, 2001.

[33] J. S. Zhang and D. P. Yang, "A splitting positive definite mixed element method for second-order hyperbolic equations," Numerical Methods for Partial Differential Equations, vol. 25, no. 3, pp. 622-636, 2009.

[34] Y. Liu, H. Li, J. F. Wang, and S. He, "Splitting positive definite mixed element methods for pseudohyperbolic equations," Numerical Methods for Partial Differential Equations, vol. 28, no. 2, pp. 670-688, 2012.

[35] Z. D. Luo, Mixed Finite Element Methods and Applications, Chinese Science Press, Beijing, China, 2006.

[36] P. G. Ciarlet, The Finite Element Methods for Elliptic Problems, North-Holland, New York, NY, USA, 1978.

[37] R. A. Adams, Sobolev Spaces, Academic Press, New York, NY, USA, 1975.

[38] H. Brunner and P. J. van der Houwen, The Numerical Solution of Volterra Equations, vol. 3, NorthHolland, Amsterdam, The Netherlands, 1986.

[39] D. Zwillinger, Handbook of Differential Equations, Academic Press, Boston, Mass, USA, 3rd edition, 1997. 
[40] F. Brezzi, J. Douglas, Jr., and L. D. Marini, "Two families of mixed finite elements for second order elliptic problems," Numerische Mathematik, vol. 47, no. 2, pp. 217-235, 1985.

[41] F. Brezzi, J. Douglas, Jr., M. Fortin, and L. D. Marini, "Efficient rectangular mixed finite elements in two and three space variables," RAIRO Modélisation Mathématique et Analyse Numérique, vol. 21, no. 4, pp. 581-604, 1987.

[42] P. A. Raviart and J. M. Thomas, "A mixed finite element method for 2nd order elliptic problems," in Mathematical Aspects of Finite Element Methods, vol. 606 of Lecture Notes in Mathematics, pp. 292-315, Springer, Berlin, Germany, 1977.

[43] Z. Chen, Finite Element Methods and Their Applications, Scientific Computation, Springer, Berlin, Germany, 2005.

[44] M. F. Wheeler, "A priori $L^{2}$-error estimates for Galerkin approximations to parabolic partial differential equations," SIAM Journal on Numerical Analysis, vol. 10, no. 4, pp. 723-749, 1973.

[45] L. Chen and Y. P. Chen, "Two-grid method for nonlinear reaction-diffusion equations by mixed finite element methods," Journal of Scientific Computing, vol. 49, no. 3, pp. 383-401, 2011. 


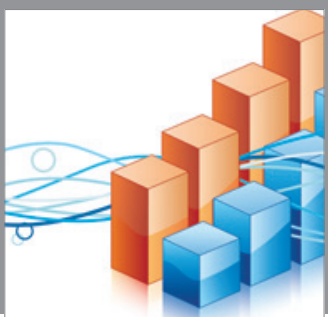

Advances in

Operations Research

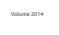

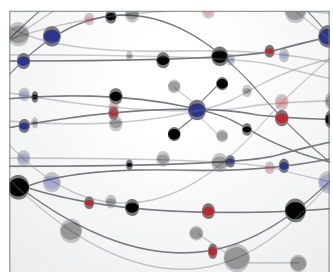

\section{The Scientific} World Journal
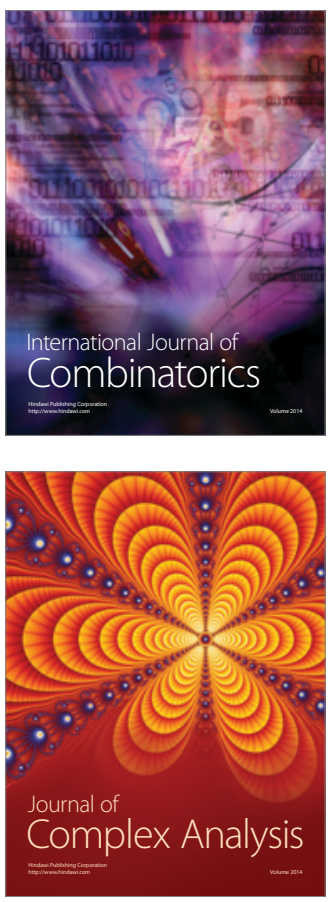

International Journal of

Mathematics and

Mathematical

Sciences
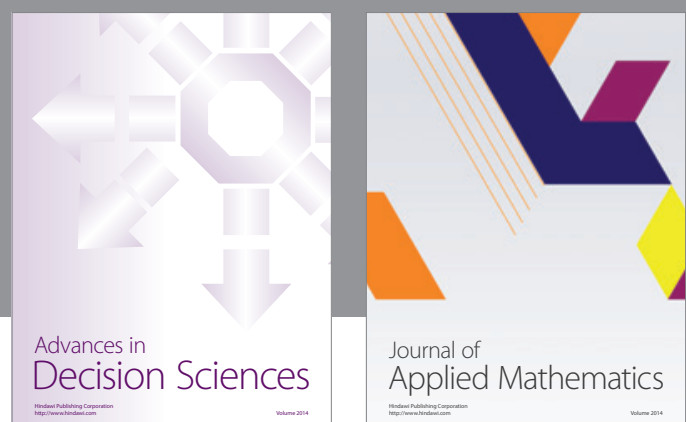

Journal of

Applied Mathematics
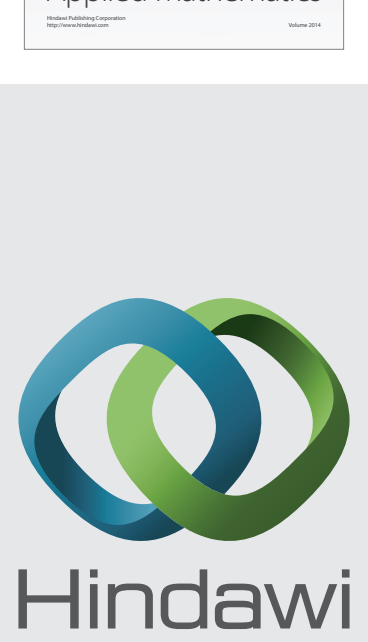

Submit your manuscripts at http://www.hindawi.com
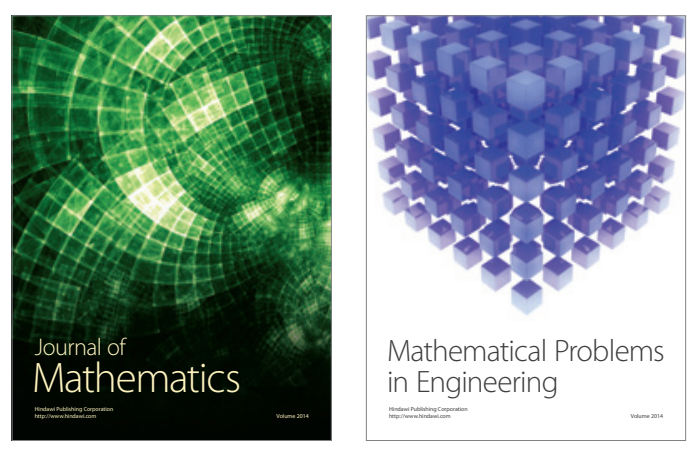

Mathematical Problems in Engineering
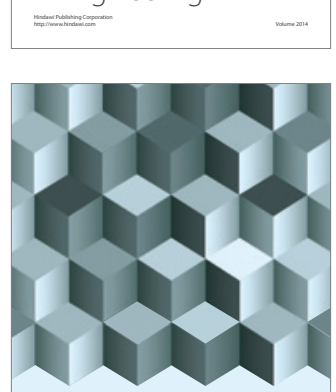

Journal of

Function Spaces
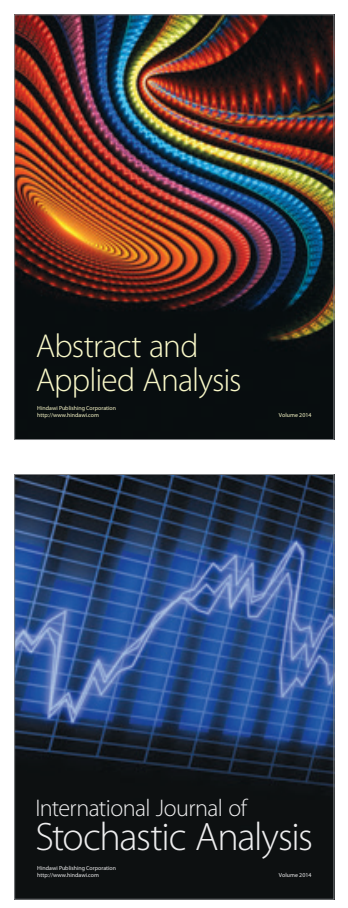

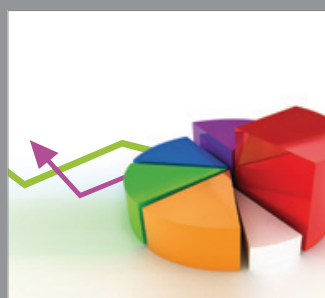

ournal of

Probability and Statistics

Promensencen
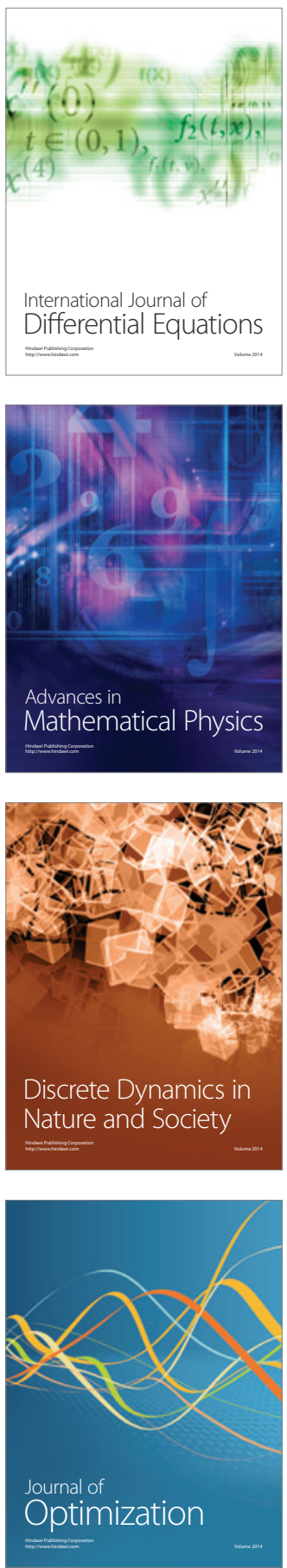